1908.] Representation of a function by series of Bessel's functions. 359

\title{
ON THE REPRESENTATION OF A FUNCTION BY SERIES OF BESSEL'S FUNCTIONS
}

\author{
$B y$ E. W. Hobson.
}

[Received and Read December 10th, 1908.]

The method of investigation of the convergence of series which represent a function of a real variable in a given interval which I have developed in my paper* "On a General Convergence Theorem, and the Theory of the Representation of a Function by Series of Normal Functions," is here applied to the case of series of Bessel's functions. A function $f(z)$ defined for the interval $(0,1)$ may be represented in that interval by a series $\sum_{n=1}^{\infty} c_{n} J_{m}\left(k_{n} z\right)$, where $m$ is a prescribed constant $\geqslant 0$, and the numbers $k_{n}$ are the positive roots of the equation

$$
k J_{m}^{\prime}(k)+H J_{m}(k)=0 .
$$

The real number $H$ may have any assigned value including the value zero, and we may regard the value $H=\infty$ as applying to the case in which the equation is

$$
J_{m}(k)=0 \text {. }
$$

The coefficients $c_{n}$ are of known form.

It is here shewn that, if the function $f(z)$ is such that $z^{\frac{1}{2}} f(z)$ has a Lebesgue integral in the interval $(0,1)$, the question whether the series converges, or not, at any interior point of the interval, or also at the endpoint 1 , depends only on the nature of the function $f(z)$ in an arbitrarily small neighbourhood of that point. It is moreover shewn that at such a point the series converges to the value $\frac{1}{2}\{f(z+0)+f(z-0)\}$, if the sufficient condition is satisfied, that a neighbourhood of the point $z$ exists in which the function is of limited total fluctuation (à variation bornée). It is further shewn that, if $f(z)$ be continuous in an interval $(a, b)$ contained in $(0,1)$, then the series converges uniformly in $(a, b)$ to the value $f(z)$ in case $(a, b)$ is contained in the interior of another interval $\left(a^{\prime}, b^{\prime}\right)$ in which the function is of limited total fluctuation; the interval $\left(a^{\prime}, b^{\prime}\right)$ may exceed $(a, b)$ in length by an arbitrarily small amount. 
The condition that $z^{\frac{1}{1}} f(z)$ should have a Lebesgue integral in the interval $(0,1)$ is satisfied if $f(z)$, whether it be limited or not, have a Lebesgue integral in the interval, provided further that $\int_{0}^{e} z^{\mathrm{b}} f(z) d z$ exists. This last condition will be satisfied, in particular, if $f(z)$ is infinite at $z=0$, and of order less than $\frac{3}{2}$, or, more generally, if any of the known sufficient conditions for the convergence of such an integral are satisfied.

The case of the series of Bessel's functions differs from that of the series of Sturm-Liouville functions considered in my former paper, on account of the fact of the existence of the singularity at the point $z=0$ of the differential equation satisfied by the Bessel's functions. It may be remarked that the series of Bessel's functions necessary for the representation of a function in an interval $(a, b)$, where $0<a<b$, and in which Bessel's functions of both kinds occur, fall under the category of series of Sturm-Liouville functions, since there is no singularity of the functions in the interval $(a, b)$. Such a mode of representation has accordingly been fully considered in the former paper.

The particular case of the series considered in the present communication which arises when $H=\infty, m=0$, has been treated* by Kneser for the case of a function which is of limited total fluctuation in the whole interval $(0,1)$, and is therefore a limited function. A study of Kneser's memoir has been of much assistance to me in the investigation which I here present.

1. The differential equation

$$
\frac{d}{d x}\left(x \frac{d y}{d x}\right)+\left(\lambda x-\frac{m^{2}}{x}\right) y=0
$$

is satisfied by the two Bessel's functions of order $m$, and of argument $\lambda^{\frac{1}{t}} x$. If we make $x^{2}$ the independent variable in the equation, instead of $x$, and then change $x^{2}$ into $x$, we see that the differential equation

$$
\frac{d}{d x}\left(4 x \frac{d y}{d x}\right)-\frac{m^{2}}{x} y+\lambda y=0,
$$

is satisfied by the two Bessel's functions $J_{m b}\left(\lambda^{3} x^{\frac{1}{2}}\right), Y_{m}\left(\lambda^{\frac{1}{2}} x^{\frac{1}{2}}\right)$.

We take as normal functions those solutions of this equation which satisfy the conditions (1) that they are finite at the singular point $x=0$,

* See his memoir, “ Die Theorie der Integralgleichungen und die Darstellung willkürlicher Funktionen in der mathematischen Physik," Math. Annalen, Vol. LxIII. 
1908.] Repregentation OF A FUNCTION BY SERIEs of Bessel's FUNCTIONS. 361

and (2) that, at the point $x=1$, the condition

$$
\frac{d y}{d x}+\frac{1}{2} H y=0
$$

is satisfied; the values of the parameter $\lambda$ being so determined that such solutions exist. Assuming that $m \geqslant 0$, we see that only Bessel's functions of the first kind are admissible, and that the values of $\lambda$ are determined by the equation

$$
\lambda^{\natural} J_{m}^{\prime}\left(\lambda^{\natural}\right)+H J_{m}\left(\lambda^{\natural}\right)=0,
$$

the normal functions being given by

$$
\phi_{n}(x)=J_{m}\left(\lambda_{n}^{\frac{1}{3}} x^{\frac{1}{b}}\right)\left\{\int_{0}^{1}\left\{J_{m}\left(\lambda_{n}^{\frac{1}{2}} x^{\frac{1}{3}}\right)\right\}^{2} d x\right\}^{-\frac{1}{2}},
$$

where $\lambda_{n}$ is the $n$-th of the roots of the equation (2) arranged in ascending order of magnitude.

It can easily be shewn that

$$
\begin{aligned}
\int_{0}^{1}\left\{J_{m}\left(\lambda^{b} x^{b}\right)\right\}^{2} d x & =\frac{2}{\lambda} \int_{0}^{\lambda} \alpha\left\{J_{m}(\alpha)\right\}^{2} d \alpha \\
& =\frac{1}{\lambda}\left\{\left(\lambda-m^{2}\right) J_{m}^{2}\left(\lambda^{b}\right)+\lambda J_{m}^{(2}\left(\lambda^{b}\right)\right\} ;
\end{aligned}
$$

it then follows that $\phi_{n}(x)$ is given by

$$
\phi_{n}(x)=J_{m}\left(\lambda_{n}^{k} x^{3}\right)\left\{\left(1-\frac{m^{2}}{\lambda}\right) J_{m}^{2}\left(\lambda^{\frac{1}{2}}\right)+J_{m}^{\prime 2}\left(\lambda^{3}\right)\right)^{-\frac{1}{2}} .
$$

It has here been assumed that zero is not one of the characteristic values of $\lambda$. When $\lambda=0$, that solution of the differential equation (1) which is finite at the point $x=0$ is of the form $A x^{3 n}$; and this can satisfy the condition

$$
\frac{d y}{d x}+\frac{1}{2} H y=0,
$$

for $x=1$, only in case $H+m=0$. In this case the corresponding normal function $\phi_{1}(x)$ is $(m+1)^{\frac{1}{2}} x^{\text {bn }}$.

In order to investigate the validity of the representation of an arbitrarily prescribed function by means of a series of the normal functions $\phi_{n}(x)$, in the interval $(0,1)$, it will be shewn that if $F\left(x^{\prime}, x, n\right)$ denote the expression

$$
\left(x x^{\prime}\right)^{\frac{1}{2}}\left\{\phi_{1}(x) \phi_{1}\left(x^{\prime}\right)+\phi_{2}(x) \phi_{2}\left(x^{\prime}\right)+\ldots+\phi_{n}(x) \phi_{n}\left(x^{\prime}\right)\right\},
$$

then $F\left(x^{\prime}, x, n\right)$ satisfies the conditions of the general convergence theorem 
given in my paper" "On a general Convergence Theorem, and on the Representation of Functions by Series of Normal Functions." The set of points there denoted by $G$ will in the present case consist of all the points of an interval $(\xi, 1)$, where $\dot{\xi}$ is an arbitrarily small fixed positive number.

2. It is necessary for the purposes of the investigation here undertaken to obtain some detailed properties of the Bessel's functions with complex argument.

The two Bessel's functions $J_{m}(x), Y_{m}(x)$, of order $m$, may be defined for all real and complex values of $x$ which do not lie on the negative part of the real axis, by means of the formulæ

$$
\begin{aligned}
& J_{1 i}(x)=\frac{x^{n}}{2^{m} \Pi(m)} ; 1-\frac{x^{2}}{2.2 m+2}+\frac{x^{4}}{2.4 .2 m+2.2 m+4}-\ldots ;, \\
& Y_{m}(x)=\pi e^{m \pi \iota} \frac{J_{-m}(x)-\cos m \pi J_{m i}(x)}{\sin 2 m \pi}
\end{aligned}
$$

where the expression $x^{i n}$ is taken to denote $r^{m}(\cos m \theta+\iota \sin m \theta) ; r$ denoting the modulus of $x$, and the argument $\theta$ being restricted to values between $-\pi$ and $\pi$. It is understood that, when $m$ is an integer, $Y_{m}(x)$ is defined as the limit of the expression in the second formula, as $m$ approaches such integral value. This definition of $Y_{m}(x)$ becomes nugatory when $m$ is half an odd integer. In this case we multiply both sides of the equation by $\cos m \pi$, and then write $Y_{m}(x)$ for $Y_{m}(x) \cos m \pi$; the new value of $Y_{m}(x)$ is then finite.

In the following investigations, the case in which $m$ is half an odd integer is included, if we remember that, throughout the work, the factor $\cos m \pi$ is to be in that case throughout rejected.

It is known that, when $m$ is real and $>-\frac{1}{2}$, and when the real part of $x$ is positive,

$$
\begin{aligned}
& Y_{m}(x)+\frac{1}{2} \iota \pi \frac{e^{m \pi \imath}}{\cos m \pi} J_{m}(x) \\
& \quad=\frac{\Pi\left(\frac{-1}{2}-m\right)}{\Pi\left(-\frac{1}{2}\right)} e^{\left(\frac{1}{2} m \pi+\frac{1}{2} \pi+x\right) \iota} \frac{1}{(2 x)^{\frac{1}{2}}} \int_{0}^{\infty} e^{-u} u^{m-\frac{1}{2}}\left(1+\frac{\iota u}{2 x}\right)^{m-\frac{1}{1}} d x \\
& Y_{m}(x)-\frac{1}{2} \iota \pi \frac{e^{m \pi}}{\cos m \pi} J_{m}(x) \\
& \quad=\frac{\Pi\left(-\frac{1}{2}-m\right)}{\Pi\left(-\frac{1}{2}\right)} e^{\left(\frac{\left.3 m \pi-\frac{1}{2} \pi-x\right) \iota}{2} \frac{1}{(2 x)^{\frac{1}{2}}} \int_{0}^{\infty} e^{-u} u^{n-\frac{1}{2}}\left(1-\frac{\iota u}{2 x}\right)^{m-\frac{1}{2}} d u .\right.}
\end{aligned}
$$


1908.] Representation of a function by sebies of Bessel's functions. 363

In order to estimate the value of the two integrals

$$
\int_{0}^{\infty} e^{-u} u^{m-\frac{1}{2}}\left(1 \pm \frac{\iota u}{2 x}\right)^{m-\frac{1}{2}} d u
$$

let it be first assumed that $m-\frac{3}{2} \geqslant 0$. Using Darboux's form of 'Taylor's theorem for functions of complex numbers, we have

$\int_{0}^{\infty} e^{-u} u^{m-\frac{b}{b}}\left(1 \pm \frac{\iota u}{2 x}\right)^{m-\frac{k}{2}} d u=\int_{0}^{\infty} e^{-u} u^{m-\frac{k}{2}}\left[1 \pm\left(m-\frac{1}{2}\right) \frac{\iota \lambda u}{2 x}\left(1 \pm \frac{\iota \iota \theta}{2 x}\right)^{m-\frac{\hbar}{2}}\right] d u$,

where $0 \leqslant \theta \leqslant 1$, and $\lambda$ is such that its modulus does not exceed unity. Since

$$
\bmod \left(1 \pm \frac{\iota \iota \theta}{2 x}\right) \leqslant 1+\frac{u}{2 r}
$$

where $r$ denotes the modulus of $x$, we see that the integral on the lefthand side differs from $\Pi\left(m-\frac{1}{2}\right)$ by an amount of which the modulus does not exceed

$$
\frac{1}{2 r}\left(m-\frac{1}{2}\right) \int_{0}^{\infty} e^{-u} u^{m+\frac{1}{2}}\left(1+\frac{u}{2 r}\right)^{m-\frac{a}{2}} d u
$$

or $\frac{1}{2 v}\left(m-\frac{1}{2}\right) \int_{0}^{\infty} e^{-u} u^{m+\frac{1}{2}}\left[1+\left(m-\frac{3}{2}\right) \frac{u}{2 v}+\ldots\right.$

$$
\left.+\frac{\left(m-\frac{3}{2}\right)\left(m-\frac{5}{2}\right) \ldots\left(m-\frac{1}{2}-s\right)}{s !}\left(\frac{u}{2 v}\right)^{s}\left(1+\frac{\theta^{\prime} u}{2 r}\right)^{m-\frac{1}{2}-s}\right] d u
$$

where $\theta^{\prime}$ is such that $0 \leqslant \theta^{\prime} \leqslant 1$.

If now $s$ be taken to be the integer next greater than $m-\frac{3}{2}$, or equal to $m-\frac{3}{2}$, we see that

$$
\left(1+\frac{\theta^{\prime} u}{2 r}\right)^{m-i-s} \leqslant 1
$$

hence the modulus does not exceed a number of the form

$$
\frac{a_{0}}{r}+\frac{a_{1}}{r^{2}}+\ldots+\frac{a_{s}}{r^{s+1}}
$$

where $a_{0}, a_{1}, \ldots, a_{s}$ are positive numbers. It has therefore been shewn that

$$
\int_{0}^{\infty} e^{-u} u^{m-\frac{1}{2}}\left(1 \pm \frac{\iota u}{2 x}\right)^{m-\frac{1}{2}} d u=\Pi\left(m-\frac{1}{2}\right) ! 1+\frac{u(x)}{r} ;
$$

where $a(x)$ denotes a number of which the modulus is less than some fixed positive number independent of $x$, for all values of $x$ of which the moduli exceed an arbitrarily chosen positive number. 
We now see that, if $m-\frac{3}{2} \geqslant 0, J_{m}(x), Y_{m}(x)$ are given by

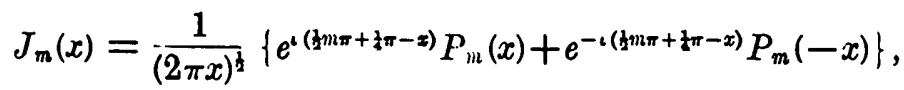

$$
\begin{aligned}
& Y_{m}(x)=\frac{\iota}{2}\left(\frac{\pi}{2 x}\right)^{!} e^{m \pi \imath} \sec m \pi\left\{-e^{\iota\left(\frac{1}{m \pi}+\xi \pi-x\right)} P_{n l}(x)+e^{-\imath\left(3 m \pi+\frac{\xi}{2} \pi-x\right)} P_{m}(-x)\right\} \text {, }
\end{aligned}
$$

where $P_{m}(x), P_{m}(-x)$ are both of the form $1+\frac{\alpha(x)}{r}$, and $\alpha(x)$ denotes two different numbers such that $|\alpha(x)|$ is less than a fixed number independent of $x$, provided $r$ is not less than some arbitrarily chosen positive number $\eta$. This result can now be extended to the case in which $m-\frac{1}{2} \geqslant 0$.

Since

$$
J_{m}(x)=\frac{2(m+1)}{x} J_{m+1}(x)-J_{m+2}(x),
$$

and

$$
Y_{m}(x)=\frac{2(m+1)}{x} Y_{m+1}(x)-Y_{m+2}(x),
$$

we find that, assuming the above expressions to hold for $m+1, m+2$, they hold also for $m$, where

$$
\begin{aligned}
P_{m}(x) & =\frac{2(m+1) \iota}{x} P_{m+1}(x)+P_{m+2}(x), \\
P_{m}(-x) & =-\frac{2(m+1) \iota}{x} P_{m+1}(-x)+P_{m+2}(-x) .
\end{aligned}
$$

It is then clear that $P_{m}(x), P_{m}(-x)$ are of the form $1+\frac{a(x)}{r}$, since $P_{m+1}(x), P_{m+2}(x), P_{m+1}(-x), P_{m+2}(-x)$ are of this form.

In an exactly similar manner it is shewn that the theorem holds if $m+\frac{1}{2} \geqslant 0$, and generally for all real values of $m$. It has been shewn that the functions $J_{n}(x), Y_{m}(x)$ are expressed by the formulæ (4), where $\left|P_{m}(x)\right|$ is less than some fixed number independent of $x$, provided $\bmod x$ is not less than an arbitrarily fixed positive number $\eta$. Moreover, $P_{m}(x)$ is of the form $1+\frac{\alpha(x)}{r}$, where $\alpha(x)$ has a similar property, subject to the same condition. The theorem has been proved on the hypothesis that $x$ has a real positive part; and from the fact that the functions are continuous on the imaginary axis it follows that the theorem also holds for values of $x$ of which the real part is zero. By using the known expressions for $J_{m}(-x), Y_{m}(-x)$ in terms of $J_{m}(x), Y_{m}(x)$, it is seen that 
1908.] Representation of a Function By SERIES of Bessel's functions. 365

the result holds for the whole plane of $x$, a section along the negative part of the real axis being excluded.

The differential coefficients of $J_{m}(x), Y_{m}(x)$ may be expressed by means of the formulæ

$$
\begin{aligned}
& J_{m}^{\prime}(x)=\frac{m}{x} J_{m}(x)-J_{m+1}(x), \\
& Y_{m}^{\prime}(x)=\frac{m}{\ddot{x}} Y_{m}(x)-Y_{m+1}(x) .
\end{aligned}
$$

It follows that, when the real part of $x$ is positive or zero,

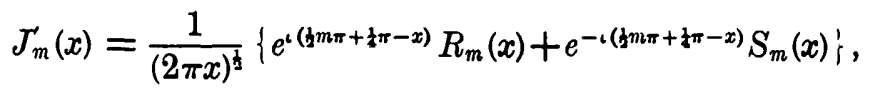

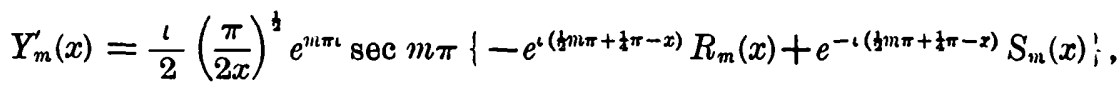

where

$$
\begin{aligned}
& R_{m}(x)=\frac{m}{x} P_{m}(x)-\iota P_{m+1}(x), \\
& S_{m}(x)=\frac{m}{x} P_{m l}(-x)+\imath P_{m+1}(x) .
\end{aligned}
$$

It is then seen that, if $\bmod x \geqslant \eta,\left|R_{m}(x)\right|,\left|S_{m}(x)\right|$ are both less than some fixed positive number independent of $x$.

Since

$$
x^{\frac{1}{1}} J_{m l}(x)=\frac{x^{m+1}}{2^{m} \Pi(m)}\left\{1-\frac{x^{2}}{2.2 m+2}+\frac{x^{4}}{2.4 .2 m+2.2 m+4}-\ldots\right\},
$$

we see that, when $m \geqslant-\frac{1}{2}$,

$$
\left|x^{3} J_{m}(x)\right|<\frac{r^{m+1}}{2^{n} \Pi \Pi(m)}\left\{1+\frac{r^{2}}{1.2}+\frac{r^{4}}{1.2 .3 .4}+\ldots\right\} .
$$

From this, it follows that, provided $m>-\frac{1}{2}$, for all values of $x$, such that $\bmod x$, or $r$, is less than an arbitrarily chosen number $\eta_{1},\left|x^{b} J_{m}(x)\right|$ is less than some fixed positive number $N_{1}$ independent of $x$.

If $x=s+\iota t$, where $t$ is positive or zero, and $s$ is positive or zero, we see from the formula (4), that

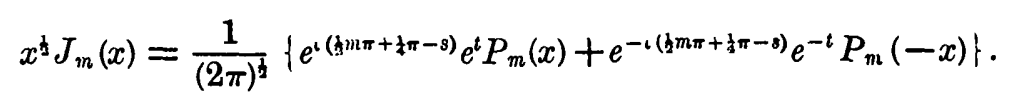

Since $\left|P_{n}(x)\right|,\left|P_{m}(-x)\right|$ are less than fixed positive numbers inde- 
pendent of $x$ for all values of $x$, such that $\bmod x \geqslant \eta$, we see that

$$
\left|x^{\frac{1}{2}} J_{m}(x)\right|<M_{1} e^{t}+M_{2} e^{-t}<\left(M_{1}+M_{2}\right) e^{t},
$$

for $\bmod x \geqslant \eta$; where $M_{1}, M_{2}$ are positive numbers independent of $x$.

It now follows that $\left|x^{\natural} J_{m}(x)\right|<N e^{\imath}$, for all values of $x(\equiv s+\iota t)$ of which the real part is positive or zero; the number $N$ being the greater of the two numbers $N_{1}, M_{1}+M_{2}$, and being therefore independent of $x, s$, and $t$.

3. The characteristic values of $\lambda$ are given as the roots of the equation

$$
\lambda^{\frac{1}{3}} J_{m}^{\prime}\left(\lambda^{\frac{1}{3}}\right)+H J_{m}\left(\lambda^{\frac{1}{3}}\right)=0,
$$

which reduces to $J_{m}^{\prime}\left(\lambda^{\frac{k}{3}}\right)=0$, in the case $H=0$, and to $J_{m n}\left(\lambda^{\frac{3}{3}}\right)=0$, in the case $H=\infty$. The value $\lambda=0$ is admissible only in the case $H=-m$, when the equation is equivalent to $J_{m+1}\left(\lambda^{b}\right)=0$. It is clear that, if $\lambda^{\frac{1}{2}}$ is a root of the equation, $-\lambda^{\frac{1}{2}}$ is also a root; and thus we need attend only to the positive roots.

We may use for $J_{m}\left(\lambda^{\frac{1}{3}}\right), J_{m}^{\prime}\left(\lambda^{\frac{1}{b}}\right)$, the expressions

$$
\begin{aligned}
& \left.\left(\frac{2}{\pi \lambda^{\frac{1}{3}}}\right)^{\frac{3}{3}} ; \cos \left(\frac{1}{2} m \pi+\frac{1}{4} \pi-\lambda^{3}\right)+\frac{\alpha(\lambda)}{\lambda^{\frac{3}{3}}}\right\}, \\
& \left(\frac{2}{\pi \lambda^{\frac{3}{3}}}\right)^{\frac{1}{2}}\left\{\sin \left(\frac{1}{2} m \pi+\frac{1}{4} \pi-\lambda^{\frac{3}{3}}\right)+\frac{\alpha(\lambda)}{\lambda^{\frac{3}{2}}}\right),
\end{aligned}
$$

where $\alpha(\lambda)$ denotes in each case some number which is less in absolute value than some fixed number independent of $\lambda$, provided $\lambda$ exceeds some fixed positive number as small as we please. The function $a(\lambda)$ does not denote the same function in the fwo cases, and it will be used throughout in this manner to denote a variety of functions which are all limited for all sufficiently large values of the variable.

We can now write the characteristic equation in the form

$$
\sin \left(\frac{1}{2} m \pi+\frac{1}{4} \pi-\lambda^{2}\right)=\lambda^{-\frac{1}{2}} a(\lambda),
$$

except that in the case $H=\infty$, it takes the form

$$
\cos \left(\frac{1}{2} m \pi+\frac{1}{4} \pi-\lambda^{\frac{1}{2}}\right)=\lambda^{-\frac{1}{2}} \alpha(\lambda) \text {. }
$$

By considering large values of $\lambda$, and then including all the values of $\lambda$ in the same expression, we see that all the values $\lambda_{1}^{k}, \lambda_{2}^{k}, \ldots, \lambda_{n}^{k}, \ldots$ of $\lambda^{b}$ are given by

$$
\lambda_{n}^{\frac{1}{2}}=\kappa+n \pi+n^{-1} \alpha(n), \text { where } \kappa=\frac{1}{2} m \pi+\frac{1}{4} \pi+s \pi,
$$

and $s$ denotes a fixed positive or negative integer. In the case $H=\infty$, 
the number $\alpha$ must be replaced by $\frac{1}{2} m \pi+\frac{3}{4} \pi+s \pi$. The number $\alpha$ is independent of $n$, and $a(n)$ is limited for all values of $n$.

Referring to the expression (3) for $\phi_{n}(x)$, we see that

$$
\left(1-\frac{m^{2}}{\lambda}\right) J_{i i l}^{2}\left(\lambda^{k}\right)+J_{m i}^{\prime 2}\left(\lambda^{3}\right)
$$

is of the form

$$
\frac{2}{\pi \lambda^{\frac{1}{2}}}\left\{1+\frac{\alpha(\lambda) !}{\lambda^{\frac{\hbar}{2}}} j,\right.
$$

and therefore $\phi_{n}(x)$ is of the form

$$
\left(\frac{1}{2} \pi \lambda^{\frac{1}{2}}\right)^{\frac{1}{2}} J_{m}\left(\lambda^{\frac{1}{a}} x^{\frac{3}{3}}\right) !\left(1+\frac{a(\lambda)}{\lambda^{\frac{1}{2}}}\right) \text {. }
$$

On substitution of the value of $J_{m}\left(\lambda^{\frac{1}{b}} x^{3}\right)$, we find for $\phi_{n}(x)$ the expression

$$
x^{-\frac{1}{2}}\left[\cos \left\{\frac{1}{2} m \pi+\frac{1}{4} \pi-(\kappa+n \pi) x^{\frac{1}{3}}\right\}+n^{-1} \alpha(n, x)\right],
$$

where $\alpha(n, x)$ is limited for all values of $n$, and for all values of $x$ in an interval $(\epsilon, 1)$, where $\epsilon$ is a positive number. An expression for $\phi_{n}(x)$ of a more detailed character, which will be required later, is obtained from the expression for $J_{m}(x)$ obtained in $\$ 2$; we find that

$$
\begin{aligned}
\phi_{n}(x)=\frac{1}{x^{3}}\left[\left\{1+\frac{a(n, x)}{n}\right\} \cos \right. & \left\{\frac{1}{2} m \pi+\frac{1}{4} \pi-(\kappa+n \pi) x^{3}\right\} \\
& \left.+\frac{a(n, x)}{n} \sin \left\{\frac{1}{2} m \pi+\frac{1}{4} \pi-(\kappa+n \pi) x^{\frac{1}{2}}\right\}\right] .
\end{aligned}
$$

4. If, in the differential equation

$$
\frac{d}{d x}\left(4 x \frac{d y}{d x}\right)+\left(\lambda-\frac{m^{2}}{x}\right) y=0
$$

we write $\lambda=\mu+\beta^{2}$, where $\beta$ is any fixed number, the differential equation becomes

$$
\frac{d}{d x}\left(4 x \frac{d y}{d x}\right)+\left(\beta^{2}-\frac{m^{2}}{x}\right) y+\mu y=0,
$$

in which we now regard $\mu$ as the parameter. The characteristic values of $\mu$ will be the values of $\lambda-\beta^{2}$, where $\lambda$ has the values determined by the equation (2), as in $\$ 3$.

In order to determine the nucleus of the integral equation for which the values of $\mu$ are the characteristic values, we take

$$
\phi(x)=C J_{m}\left(\beta x^{\natural}\right), \quad \psi(x)=A J_{m}\left(\beta x^{\natural}\right)+B Y_{m}\left(\beta x^{\natural}\right),
$$

two independent solutions of the differential equation

$$
\frac{d}{d x}\left(4 x \frac{d y}{d x}\right)+\left(\beta^{2}-\frac{m^{2}}{x}\right) y=0 ;
$$


where the constants $A, B, C$ are so determined * that

$$
4 x\left\{\phi^{\prime}(x) \psi(x)-\phi(x) \psi^{\prime}(x)\right\}=1,
$$

and that

$$
\psi^{\prime}(x)+\frac{1}{2} H \psi(x)=0,
$$

when $x=1$. The first of these equations is equivalent to

$$
2 \beta x^{\natural} B C\left\{J_{m}^{\prime}\left(\beta x^{\natural}\right) Y_{m}\left(\beta x^{\natural}\right)-J_{m}\left(\beta x^{\natural}\right) Y_{m}^{\prime}\left(\beta x^{\natural}\right)\right\}=1,
$$

and this reduces, by using the known relation between the two Bessel's functions and their derivatives, to $2 B C=e^{-m \pi} \cos m \pi$. The other equation is

$$
A\left\{\beta J_{n}^{\prime}(\beta)+H J_{n}(\beta)\right\}+B\left\{\beta Y_{m}^{\prime}(\beta)+H Y_{m}(\beta)\right\}=0,
$$

and thus we may take

$$
A=\beta Y_{m}^{\prime}(\beta)+H Y_{m}(\beta), \quad B=-\left\{\beta J_{m}^{\prime}(\beta)+H J_{m}(\beta)\right\},
$$

and therefore we have $C=-\frac{\frac{1}{2} e^{-m \pi t} \cos m \pi}{\beta J_{m}^{\prime}(\beta)+H J_{m}(\beta)}$.

The value of the nucleus $K\left(x, x^{\prime}\right)$, for $x \leqslant x^{\prime}$ is $\phi(x) \psi\left(x^{\prime}\right)$ or

$$
\begin{aligned}
\frac{-\frac{1}{2} e^{m m} \cos m \pi}{\beta J_{m}^{\prime}(\beta)+H J_{m}(\beta)} J_{m}\left(\beta x^{3}\right)\left[\beta\left\{J_{m}\left(\beta x^{\prime 3}\right) Y_{m}^{\prime}(\beta)-Y_{m}\left(\beta x^{\prime 3}\right) J_{m}^{\prime}(\beta)\right\}\right. \\
\left.+H\left\{J_{m}\left(\beta x^{\prime 3}\right) Y_{m}(\beta)-Y_{m}\left(\beta x^{\prime 3}\right) J_{m}(\beta)\right\}\right] .
\end{aligned}
$$

In case $x^{\prime} \leqslant x$, an interchange of $x$ with $x^{\prime}$ must be made in the expression.

It will now be shewn that, for all values of $x$ and $x^{\prime}$ in any interval $(\epsilon, 1)$ which does not contain the point $x=0$, the series

$$
\sum_{n=1}^{\infty} \frac{\phi_{n}(x) \phi_{n}\left(x^{\prime}\right)}{\mu_{n}} \text { or } \sum_{n=1}^{\infty} \frac{\phi_{n}(x) \phi_{n}\left(x^{\prime}\right)}{\lambda_{n}-\beta^{2}}
$$

converges uniformly to the value $K\left(x, x^{\prime}\right)$. The number $\beta$ is supposed to have been so chosen that $\mu_{n}$ is, for every value of $n$, different from zero. This result does not follow immediately from the fundamental theorem of the theory of integral equations for the representation of the nucleus, because, as is seen from the expression $(8), K\left(x, x^{\prime}\right)$ is not continuous for $x^{\prime}=0$. It has, however, been shewn by Kuezer + that, in order to establish it, we have only to verify (1) that the series

$$
\sum_{n=1}^{\infty} \frac{\phi_{n}(x) \phi_{n}\left(x^{\prime}\right)}{\mu_{n}^{2}}
$$

- See Kneser, Math. Annalen, Voi. Lxirr., p. 483.

$\dagger$ Ibid., pp. 487-489. 
converges uniformly for all values of $x$ and $x^{\prime}$ in the interval $(0,1)$, and (2) that the series

$$
\sum_{n=1}^{\infty} \frac{\phi_{n}^{\prime}(x) \phi_{n}\left(x^{\prime}\right)}{\mu_{n}^{2}}, \quad \sum_{n=1}^{\infty} \frac{\phi_{n}(x) \phi_{n}\left(x^{\prime}\right)}{\mu_{n}}
$$

are both uniformly convergent for all values of $x$ and $x^{\prime}$ in any interval $(\epsilon, 1)$, where $\epsilon>0$.

To shew that the condition (1) is satisfied, we see from the expression for $\phi_{n}(x)$, obtained in $\S 3$, that the series is of the form

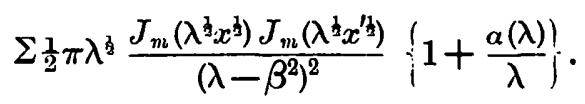

Since $J_{m}\left(\lambda^{\frac{1}{b}} x^{3}\right), J_{m}\left(\lambda^{\frac{1}{3}} x^{3}\right)$ are limited functions for all real positive values of $\lambda^{\natural}, x$, and $x^{\prime}$; and, since $\lambda$ is of the form $\left\{\kappa+n \pi+n^{-1} a(n)\right\}^{2}$ or $n^{2} \pi^{2}\left\{1+n^{-1} \alpha(n)\right\}$, it is easily seen that the series converges uniformly for all values of $x$ and $x^{\prime}$ in the interval $(0,1)$, for any fixed value of $\beta$ which does not coincide with a characteristice value of $\lambda^{\frac{1}{3}}$.

To shew that the series $\sum_{n=1}^{\infty} \frac{\phi_{n}(x) \phi_{n}\left(x^{\prime}\right)}{\mu_{n}}$ is uniformly convergent for all values of $x$ and $x^{\prime}$ in an interval $(\epsilon, 1)$, where $\epsilon>0$, we see from the the expression (6), for $\phi_{n}(x)$, that the series takes the form

$$
\begin{aligned}
\frac{1}{\left(x x^{\prime}\right)^{\frac{1}{2}}} \sum_{n=1}^{\infty} \frac{1}{n^{2} \pi^{2}}\left\{1+\frac{a(n)}{n}\right\} & {\left[\cos \left\{\frac{1}{2} m \pi+\frac{1}{4} \pi-(\kappa+n \pi) x^{3}\right\}+\frac{\alpha(n, x)}{n}\right] } \\
& {\left[\cos \left\{\frac{1}{2} m \pi+\frac{1}{4} \pi-(\kappa+n \pi) x^{\prime 3} ;+\frac{\alpha\left(n, x^{\prime}\right)}{n}\right] .\right.}
\end{aligned}
$$

On account of the factors $\frac{1}{n^{2}}, \frac{1}{n^{3}}, \frac{1}{n^{4}}$, in the different parts of the general term of the series, it is clear that the series is uniformly convergent.

To shew that the series $\sum_{n=1}^{\infty} \frac{\phi_{n}^{\prime}(x) \phi_{n}\left(x^{\prime}\right)}{\mu_{n}^{2}}$ is uniformly convergent for all values of $x$ and $x^{\prime}$ in the interval $(\epsilon, 1)$, we see that the series is of the form

$$
\begin{aligned}
\frac{1}{2 x^{3} x^{2}} \sum_{n=1}^{\infty} \frac{1}{n^{3} \pi^{3}}\left\{1+\frac{\alpha(n)}{n}\right\} & {\left[\sin \left\{\frac{1}{2} m \pi+\frac{1}{4} \pi-(\kappa+n \pi) x^{3}\right\}+\frac{u(n, x)}{n}\right] } \\
& {\left[\cos \left\{\frac{1}{2} m \pi+\frac{1}{4} \pi-(\kappa+n \pi) x^{\prime 3}\right\}+\frac{a\left(n, x^{\prime}\right)}{n}\right] ; }
\end{aligned}
$$

it is clear that this series is uniformly convergent on account of the factors $\frac{1}{n^{8}}, \frac{1}{n^{4}}, \ldots$ in the different parts of the general term.

SER. 2. VOL. 7. NO. 1028. 
It has now been established that, for any fixed real value of $\beta$, which is not equal to one of the characteristic values of $\lambda^{3}$, the series

$$
\sum_{n=1}^{\infty} \frac{\phi_{n}(x) \phi_{n}\left(x^{\prime}\right)}{\lambda_{n}-\beta^{2}}
$$

converges to the value $K\left(x, x^{\prime}\right)$ for all values of $x$ and $x^{\prime}$ in the interval $(0,1)$ except the value zero.

5. From the fact that the series $\sum_{n=1}^{\infty} \frac{\phi_{n}(x) \phi_{n}\left(x^{\prime}\right)}{\lambda_{n}-\beta^{2}}$ converges to the value $K\left(x, x^{\prime}\right)$ for any real value of $\beta$ such that $\beta^{2}$ is not equal to $\lambda_{n}$ for any value of $n$, we infer that, for complex values of $\beta$ of which the real part is positive, the series also converges to the value

$\frac{-\frac{1}{2} e^{m \pi t} \cos m \pi}{\beta J_{m}^{\prime}(\beta)+H J_{m}(\beta)} J_{m}\left(\beta x^{3}\right)\left[\beta\left\{J_{m}\left(\beta x^{\prime 3}\right) Y_{m}^{\prime}(\beta)-Y_{m}\left(\beta x^{\prime 3}\right) J_{m}^{\prime}(\beta)\right\}\right.$

provided $x \leqslant x^{\prime}$.

$$
\left.+H\left\{J_{m}\left(\beta x^{\prime 3}\right) Y_{m}(\beta)-Y_{m}\left(\beta x^{\prime 3}\right) J_{m}(\beta)\right\}\right]
$$

For a value of $\beta$ on the negative side of the imaginary axis, the series converges to the same value as for the value $-\beta$. The series

$$
\sum_{n=1}^{\infty} \phi_{r}(x) \phi_{r}\left(x^{\prime}\right)\left(\frac{1}{\beta+\lambda_{n}^{\frac{a}{n}}}+\frac{1}{\beta-\lambda_{n}^{\frac{b}{k}}}\right)
$$

consequently represents the function $-2 \beta K\left(x, x^{\prime}\right)$, for $0<x<x^{\prime}$, and for all values of $\beta$ of which the real part is positive or zero. By the theory of residues we see that $2 \sum_{r=1}^{r=n} \phi_{r}(x) \phi_{r}\left(x^{\prime}\right)$ is equal to $\frac{1}{2 \pi \iota}$ times the integral taken round a closed contour which includes in its interior the $2 n$ points $\pm \lambda_{1}^{\frac{\hbar}{2}}, \pm \lambda_{2}^{\frac{j}{2}}, \ldots, \pm \lambda_{n}^{\frac{\hbar}{j}}$ of the function $F(\beta)$ which is represented by $-2 \beta K\left(x, x^{\prime}\right)$ for values of $\beta$ of which the real part is positive or zero, and which is such that $F(-\beta)=-F(\beta)$. Taking as the path of integration the rectangle of which the corners are the four points $\pm \sigma \pm \imath \tau$, and which encloses exactly $2 n$ of the characteristic points $\pm \lambda^{k}$, and making use of the fact that conjugate values of $F(\beta)$ correspond to conjugate values of $\beta$, and that $F(-\beta)=-F(\beta)$, we see that

$$
\sum_{r=1}^{r=n} \phi_{r}(x) \phi_{r}\left(x^{\prime}\right)
$$

is equal to the real part of

$$
\frac{1}{2 \pi} \int_{0} F(\sigma+\iota t) d t-\frac{1}{2 \pi \iota} \int_{0}^{\sigma} F(s+\iota \tau) d s .
$$

Thence we see that

$$
\left|\sum_{r=1}^{r=n} \phi_{r}(x) \phi_{r}\left(x^{\prime}\right)\right|
$$


1908.] Representation of a FUnCtion By ge Ries of Bessel's functions. 371

is less than $\frac{1}{2 \pi} \int_{0}|F(\sigma+\iota t)| d t+\frac{1}{2 \pi} \int_{0}^{\sigma}|F(s+\iota \tau)| d s$,

where $F(\beta)$ denotes the expression

$$
\begin{aligned}
& \frac{-e^{m \pi \imath} \cos m \pi}{\beta J_{m}^{\prime}(\beta)+H J_{m}(\beta)} \beta J_{m}\left(\beta x^{\frac{1}{2}}\right)\left[\beta \left\{J_{m}\left(\beta x^{\prime 3}\right)\right.\right.\left.Y_{m}^{\prime}(\beta)-Y_{m}\left(\beta x^{\prime 3}\right) J_{m}^{\prime}(\beta)\right\} \\
&+H\left\{J_{m}\left(\beta x^{\prime 2}\right) Y_{m}(\beta)-Y_{m}\left(\beta x^{\prime \prime}\right) J_{m}(\beta)_{i}^{\prime}\right]
\end{aligned}
$$

Let us now assume that $\sigma$ is chosen to be $\kappa+n \pi+\frac{1}{2} \pi, n$ being chosen so large that $n^{-1} \alpha(n)$ in the expression $\kappa+n \pi+n^{-1} \alpha(n)$ for $\lambda_{n}^{j}$ is numerically less than $\frac{1}{2} \pi$. The side of the rectangle thus avoids the neighbourhood of the point $\lambda_{n}^{\frac{1}{b}}$.

We have now, for $\beta=\sigma+\iota t$, where $t \geqslant 0$,

$$
\begin{aligned}
(2 \pi \beta)^{\natural}\left\{J_{m}^{\prime}(\beta)+\frac{H}{\beta} J_{m}(\beta)\right\}= & \pm \iota e^{t}\left[\frac{m}{\beta} P_{m}(\beta)-\iota P_{m+1}(\beta)\right] \\
& \mp \iota e^{-t}\left[\frac{m}{\beta} P_{m}(-\beta)+\iota P_{m+1}(\beta)\right] \\
& \pm \iota e^{t} P_{m}(\beta) \pm \iota e^{-t} \frac{H}{\beta} P_{m}(-\beta) \\
= & \pm e^{t}\left[P_{m+1}(\beta)+\imath \frac{H+m}{\beta} P_{m}(\beta)\right] \\
& \pm e^{-t}\left[P_{m+1}(\beta)+\iota \frac{H-m}{\beta} P_{m}(-\beta)\right] .
\end{aligned}
$$

It has been shewn, in $\S 2$, that $P_{m+1}(\beta)$ has the limit unity as $|\beta|$ is indefinitely increased; we thus see that, for a sufficiently large value of $\beta$, the expression on the right-hand side has a real part of the form $\pm e^{t}(1+\eta) \pm e^{-t}(1+\xi)$, where $\eta$ and $\xi$ are numerically less than an arbitrarily chosen number as small as we please. It follows that

$$
\left|(2 \pi \beta)^{\frac{1}{2}}\left\{J_{m}^{\prime}(\beta)+\frac{H}{\beta} J_{m}(\beta)\right\}\right|
$$

exceeds

$$
e^{t}\left(1+e^{-2 t}+\eta+\xi e^{-2 \eta}\right) \text {. }
$$

If, now, $\sigma$ exceed some fixed value, we shall have $\left|\eta+\xi e^{-2 t}\right|<\frac{1}{2}$ for for all positive values of $t$; it then follows that

$$
\left|(2 \pi \beta)^{\frac{1}{2}}\left\{J_{m}^{\prime}(\beta)+\frac{H}{\beta} J_{m}(\beta)\right\}\right|>\frac{1}{2} e^{t} .
$$


In the case $H=\infty$, we can shew in a similar manner that

$$
\left|(2 \pi \beta)^{\frac{1}{b}} J_{m}(\beta)\right|>\frac{1}{2} e^{t},
$$

for all positive values of $t$, provided $\sigma$ is sufficiently large.

When we take $\beta=s+i \tau$, where $\tau$ is fixed, and $s$ varies between 0 and $\sigma$, we have

$$
\begin{aligned}
& (2 \pi \beta)^{\frac{1}{t}}\left\{J_{m}^{\prime}(\beta)+\frac{H}{\beta} J_{m}(\beta)\right\}=e^{\tau} e^{\iota\left(\hat{z} m \pi+\frac{1}{t}-s\right)}\left[\frac{m}{\beta} P_{m}(\beta)-\iota P_{m+1}(\beta)\right] \\
& +e^{-\tau} e^{-\imath\left(3 m \pi+\frac{1}{2} \pi-s\right)}\left[\frac{m}{\beta} P_{m}(-\beta)+\iota P_{m+1}(\beta)\right]
\end{aligned}
$$

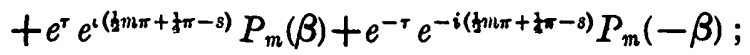

hence we find that

$$
(2 \pi \beta)^{\frac{1}{2}}\left\{J_{m}^{\prime}(\beta)+\frac{H}{\beta} J_{m}(\beta)\right\}=A e^{\tau}+B e^{-\tau},
$$

where $|A|,|B|$ can be made as nearly equal to unity as we please by taking a large enough value of $\tau$. Also $|B|$ is less than some fixed positive number $p$, if $|\beta|$ exceeds a fixed value. We thus see that

$$
\left|(2 \pi \beta)^{\frac{1}{2}}\left\{J_{m}^{\prime}(\beta)+\frac{H}{\beta} J_{m}(\beta)\right\}\right|>\frac{1}{2} e^{\tau}-p e^{-\tau},
$$

for all values of $s$, provided $\tau$ is sufficiently large. If now $\tau$ be further increased, if necessary, so that $p e^{-2 \tau}<\frac{1}{4}$, we see that

$$
\left|(2 \pi \beta)^{\frac{1}{2}}\left\{J_{m}^{\prime}(\beta)+\frac{H}{\beta} J_{m}(\beta)\right\}\right|>\frac{1}{4} e^{\tau},
$$

provided $\tau$ have a sufficiently large value; and this holds for all positive values of $s$.

In the case $H=\infty$, we can prove in a similar manner that

$$
\left|(2 \pi \beta)^{\frac{1}{b}} J_{m}(\beta)\right|>\frac{1}{4} e^{\tau},
$$

for all sufficiently large values of $\tau$, and for all positive values of $s$.

An inferior limit for the values of

$$
\left|(2 \pi \beta)^{\frac{1}{2}}\left\{J_{m}^{\prime}(\beta)+\frac{H}{\beta} J_{m}(\beta)\right\}\right|,
$$

or in the case $H=\infty$, of $\left|(2 \pi \beta)^{\frac{1}{3}} J_{m}(\beta)\right|$, has now been found for its values as they occur in the integrands of the two integrals

$$
\int_{0}^{\tau}|F(\sigma+\iota t)| d t, \quad \int_{0}^{\sigma}|F(s+\imath \tau)| d s
$$


provided the values of $\sigma$ and $\tau$ are subject to the conditions indicated above.

It has been shewn in $\$ 2$ that $\left|\beta^{h} x^{\frac{1}{4}} J_{m}\left(\beta x^{\natural}\right)\right|<N e^{t, 1}$, where $\beta=s+\iota t$, and $N$ is independent of $\beta$ and of $x$, and $0 \leqslant x \leqslant 1$. Let it now be assumed that $x^{\prime}-x \geqslant \mu$, where $\mu$ is some fixed positive number; we have then

$$
x^{\prime \frac{1}{2}}-x^{\frac{1}{2}} \geqslant \frac{\mu}{x^{1 / 2}+x^{\frac{1}{2}}} \geqslant \frac{1}{2} \mu .
$$

We now see that, on substitution of the values of the functions given in $\S 2$,

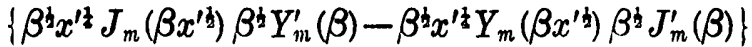

$$
\begin{aligned}
& +\frac{H}{\beta}\left\{\beta^{\natural} x^{\prime \downarrow} J_{m}\left(\beta x^{\prime b}\right) \beta^{\natural} Y_{m}(\beta)-\beta^{h} x^{\prime t} Y_{m}\left(\beta x^{\prime b}\right) \beta^{\natural} J_{m}(\beta)_{i}^{\prime}\right.
\end{aligned}
$$

takes the form

$$
\begin{aligned}
& \frac{1}{2}\left(e^{m \pi \imath} \sec m \pi\left[e^{i \beta\left(1-x^{\natural}\right)} P_{m}\left(\beta x^{\prime 3}\right) S_{m}(\beta)-e^{-\imath \beta\left(1-x^{\prime}\right)} P_{m}\left(-\beta x^{\prime \xi}\right) R_{m}(\beta)\right]\right. \\
& +\frac{H}{\beta} \frac{1}{2} e^{m \pi \imath} \sec m \pi\left[e^{i \beta\left(1-x^{\prime}\right)} P_{m}\left(\beta x^{\prime k}\right) P_{m}(-\beta)-e^{-i \beta\left(1-x^{\prime}\right)} P_{m l}\left(-\beta x^{\prime}\right) P_{m}(\beta)\right] \text {. }
\end{aligned}
$$

Since $\left|\beta x^{\prime \prime}\right|$ is always greater than some fixed positive number, in consequence of the fact that $x^{\prime \frac{1}{2}}>\frac{1}{2} \mu$, and since $\left|P_{n}\left( \pm \beta x^{\prime b}\right)\right|,\left|R_{m}(\beta)\right|$, $\left|S_{m}(\beta)\right|$ are, as has been shewn in $\S 2$, all less than fixed positive numbers independent of $\beta$ and $x^{\prime}$, we see that, for $\beta=s+\iota t$, the modulus of the expression is less than $A e^{\left(1-x^{d}\right) t}+B e^{-\left(1-x^{\natural}\right) t}$, where $A$ and $B$ are positive numbers independent of $x^{\prime}$ and $\beta$. It follows that the modulus of the expression is less than $K e^{\left(1-x^{4}\right) \ell}$, where $K=A+B$. In the case $H=\infty$, we can prove, in a similar manner, that

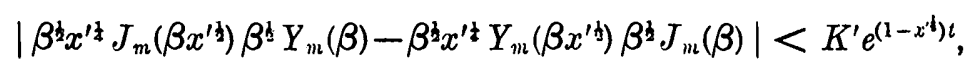

where $K^{\prime}$ is a positive number, independent of $x^{\prime}$ and $\beta$.

We have now, by the use of the inequalities which have been established,

$$
\left|\left(x x^{\prime}\right)^{ \pm} \sum_{r=1}^{r=n} \phi_{r}(x) \phi_{r}\left(x^{\prime}\right)\right|<L \int_{0}^{r} e^{-\left(x^{4}-x^{d}\right) t} d t+M \sigma e^{-\left(x^{3}-x^{3}\right) \tau},
$$

where $L$ and $M$ are fixed positive numbers. This has been proved on the assumption that $n$ is greater than some fixed value $n_{1}$, so that the values of $\sigma$ and $\tau$ are sufficiently great for the validity of the inequalities employed. If $\sigma$ have a fixed value, we may choose in value of $\tau$, independent of $x$ and $x^{\prime}$, such that $M \sigma \sigma e^{-\left(,^{\prime 4}-x^{3}\right) \tau}$ is less than $n$ tixed positive number $m$, since $x^{\prime 3}-x^{1}>\frac{1}{2} \mu$. 
Also, since $\quad \int_{0} e^{-\left(x^{4}-x^{4}\right) t} d t=\frac{1-e^{-\left(x^{4}-x^{4}\right)}}{x^{1 / 4}-x^{\frac{1}{3}}}<\frac{2}{\mu}$,

we see that

$$
L \int_{0}^{\tau} e^{-\left(x^{4}-x^{4}\right) t} d t<\frac{2 L}{\mu} .
$$

It follows that, if $n>n_{1}$,

$$
\left|\left(x x^{\prime}\right)^{ \pm} \sum_{r=1}^{r=n} \phi_{r}(x) \phi_{r}\left(x^{\prime}\right)\right|<m+\frac{2 L}{\mu},
$$

which is independent of $x$ and $x^{\prime}$. It has been assumed that $x>0$, $x^{\prime}-x \geqslant \mu$; but the inequality clearly holds also for $x=0, x^{\prime}-x \geqslant \mu$, since

$$
\left|\left(x x^{\prime}\right)^{t} \sum_{r=1}^{r=n} \phi_{r}(x) \phi_{r}\left(x^{\prime}\right)\right|
$$

vanishes when $x=0$. As the number of values of the expression for $n \leqslant n_{1}$ is finite, it follows that, for all values of $n$,

$$
\left|\left(x x^{\prime}\right)^{\frac{1}{d}} \sum_{r=1}^{\prime=1} \phi_{r}(x) \phi_{r}\left(x^{\prime}\right)\right|
$$

is less than some fixed positive number, independent of $x$ and $x^{\prime}$, for all values of $x$ and $x^{\prime}$ in the interval $(0,1)$ and such that $x^{\prime}-x \geqslant \mu$. Since $x, x^{\prime}$ may be interchanged, the last condition may be replaced by $\left|x^{\prime}-x\right| \geqslant \mu$.

6. In order to shew that the general convergence theorem already referred to is applicable to the function

$$
F\left(x^{\prime}, x, n\right)=\left(x: x^{\prime}\right)^{\ddagger} \sum_{r=1}^{r=n} \phi_{r}(x) \phi_{r}\left(x^{\prime}\right),
$$

it is necessary to shew that, if $x$ be now confined to an interval $(\xi, 1)$, where $0<\xi<1$, and if $a, \beta$ are in the interval $(0,1), \int_{a}^{\beta} F\left(x^{\prime}, x, n\right) d x^{\prime}$ is numerically less than a tixed positive number $A_{n}$, independent of $\alpha, \beta$, and $x$, provided $x$ is not interior to the interval $(\alpha-\mu, \beta+\mu)$, and is in the interval $(\xi, 1)$, all the points of which then constitute the set $G$ of the general convergence theorem. This is equivalent to shewing that, if $\epsilon$ be a fixed positive number, arbitrarily small, a value $n_{1}$ of $n$ can be determined such that

$$
\left|\int_{a}^{\beta} F\left(x^{\prime}, x, n\right) d x^{\prime}\right|<\epsilon,
$$

for every value of $n \geqslant n_{1}$, for all values of $\alpha, \beta$ in $(0,1)$, and for all values of $x$ in $(\xi, 1)$ and not interior to the interval $(\alpha-\mu, \beta+\mu)$. 
1908.] Representation of a function By geries of Bessel's functions. 375

Since

$$
\left|\left(x x^{\prime}\right)^{\frac{3}{4}} \sum_{r=1}^{r=n} \phi_{r}(x) \phi_{r}\left(x^{\prime}\right)\right|<\vec{F},
$$

if $\left|x-x^{\prime}\right| \geqslant \mu$, we see that, if $\eta<\frac{\epsilon}{2 \bar{F}}$,

then

$$
\left|\int_{0}^{n} F\left(x^{\prime}, x, n\right) d x^{\prime}\right|<\frac{1}{2} \epsilon,
$$

for all values of $n$, and for all values of $x \geqslant \eta+\mu$. It is clear that $\eta$ can be so chosen as to be less than $\mu$. In case $0 \leqslant \alpha<\eta<\beta$, we have

$$
\left|\int_{a}^{\beta} F\left(x^{\prime}, x, n\right) d x^{\prime}\right|<\frac{1}{2} \epsilon+\left|\int_{\eta}^{\beta} F\left(x^{\prime}, x, n\right) d x^{\prime}\right| ;
$$

and, if $0 \leqslant \alpha<\beta<\eta$, we have

$$
\left|\int_{a}^{\beta} F\left(x^{\prime}, x, n\right) d x^{\prime}\right|<\frac{1}{2} \epsilon,
$$

for all values of $n$. It will therefore be sufficient to prove that

$$
\left|\int_{a}^{\beta} F\left(x^{\prime}, x, n\right) d x^{\prime}\right|<\frac{1}{2} \epsilon,
$$

for $n \geqslant n_{1}$, where $\alpha$ is now restricted to be $\geqslant \eta$.

Using Bonnet's form of the second mean value theorem, we see that

$$
\int_{a}^{\beta}\left(x x^{\prime}\right)^{\frac{1}{r}} \sum_{r=1}^{r=n} \phi_{r}(x) \phi_{r}\left(x^{\prime}\right) d x^{\prime}=(x \beta)^{\ddagger} \int_{\gamma}^{\beta} \sum_{r=1}^{r=n} \phi_{r}(x) \phi_{r}\left(x^{\prime}\right) d x^{\prime},
$$

where $\gamma$ is some number such that $a \leqslant \gamma \leqslant \beta$. Since $(x \beta)^{\frac{1}{1}}<1$, it will be sufficient for our purpose if we prove that

$$
\left|\int_{\alpha^{\prime}}^{\beta^{\prime}} r=1=n \phi_{r}^{r=n}(x) \phi_{r}\left(x^{\prime}\right) d x^{\prime}\right|<\frac{1}{2} \epsilon,
$$

for

$$
n \geqslant n_{1}, \quad \alpha^{\prime} \geqslant \eta, \quad\left|x-x^{\prime}\right| \geqslant \mu, \quad \xi \leqslant x \leqslant 1 .
$$

By using the differential equation satisfied by the functions $\phi_{r}(x)$, we have

$$
\begin{aligned}
& \int_{a^{\prime}}^{\beta^{\prime}} \sum_{r=1}^{r=n} \phi_{r}(x) \phi_{r}\left(x^{\prime}\right) d x^{\prime} \\
&=\int_{a^{\prime}}^{\beta^{\prime}} \sum_{r=1}^{r=n} \frac{\phi_{r}(x)}{\mu_{r}} {\left[\left(\frac{m^{2}}{x^{\prime}}-\beta^{2}\right) \phi_{r}\left(x^{\prime}\right)-\frac{d}{d x^{\prime}}\left\{4 x^{\prime} \phi_{r}^{\prime}\left(x^{\prime}\right) ;\right] d x^{\prime}\right.} \\
&=\int_{a^{\prime}}^{\beta^{\prime}}\left(\frac{m^{2}}{x^{\prime}}-\beta^{2}\right) \sum_{r=1}^{r=n} \frac{\phi_{r}(x) \phi_{r}\left(x^{\prime}\right)}{\mu_{r}} d x^{\prime} \\
&-\left\{4 \beta^{\prime} \sum_{r=1}^{r=n} \frac{\phi_{r}(x) \phi_{r}^{\prime}(\beta)}{\mu_{r}}-4 \alpha^{\prime} \sum_{r=1}^{r=n} \frac{\left.\phi_{r}(x) \phi_{r}^{\prime}\left(a^{\prime}\right)\right\}}{\mu_{r}} ;,\right.
\end{aligned}
$$

wher e $\beta$ dənotes a fixed number, as in $\$ 4$, and $\mu_{r}=\lambda_{r}-\beta^{2}$. 
It has already been shewn that, if $x^{\prime}$ is in the interval $(\eta, 1)$, and $x$ in the interval $(\xi, 1), \sum_{r=1}^{r=n} \frac{\phi_{r}(x) \phi_{r}\left(x^{\prime}\right)}{\mu_{r}}$ converges uniformly, as $n$ is indefinitely increased, to the value $K\left(x, x^{\prime}\right)$. It will here be shewn that, for all such values of $x$ and $x^{\prime}$, which also satisfy the condition $\left|x-x^{\prime}\right| \geqslant \mu$, the series $\sum_{r=1}^{r=n} \frac{\phi_{r}(x) \phi_{r}^{\prime}\left(x^{\prime}\right)}{\mu_{r}}$ converges, as $n$ is indefinitely increased, uniformly to the value $\frac{d K\left(x, x^{\prime}\right)}{d x^{\prime}}$.

The series $\sum_{r=1} \frac{\phi_{r}(x) \phi_{r}^{\prime}\left(x^{\prime}\right)}{\mu_{r}}$ is of the form $\frac{1}{\left(x x^{\prime}\right)^{\frac{3}{3}}} \frac{1}{2 \pi x^{1 / 3}} \sum_{r=1} \frac{1}{r}\left\{1+\frac{a(r)}{r}\right\}$

$$
\begin{aligned}
& {\left[\cos \left(\frac{1}{2} m \pi+\frac{1}{4} \pi\right) \cos (\kappa+r \pi) x^{\frac{1}{2}}+\sin \left(\frac{1}{2} m \pi+\frac{1}{4} \pi\right) \sin (\kappa+r \pi) x^{\frac{1}{3}}+\frac{u(r)}{r}\right]} \\
& {\left[\sin \left(\frac{1}{2} m \pi+\frac{1}{4} \pi\right) \cos (\kappa+r \pi) x^{\prime 3}-\cos \left(\frac{1}{2} m \pi+\frac{1}{4} \pi\right) \sin (\kappa+r \pi) x^{\prime 3}+\frac{\alpha(r)}{r}\right] .}
\end{aligned}
$$

Of the different portions into which this series may be split up, the series

$$
\Sigma \frac{1}{r} \cos (r \pi+\kappa)\left(x^{3} \pm x^{\prime 3}\right), \quad \Sigma \frac{1}{r} \sin (r \pi+\kappa)\left(x^{3} \pm x^{\prime 3}\right)
$$

are uniformly convergent, since $0<\frac{1}{2} \mu \leqslant\left|x^{\frac{1}{1}} \pm x^{\prime 3}\right| \leqslant 2-\frac{1}{2} \mu$. The remaining series formed by multiplying out the above product are uniformly convergent in the whole interval $(0,1)$. It follows that $\sum_{r=1}^{r} \frac{\phi_{r}(x) \phi_{\underline{r}}^{\prime}\left(x^{\prime}\right)}{\mu_{r}}$ is uniformly convergent for all values of $x^{\prime}$ in the interval $(\eta, 1)$, and of $x$ in $(\dot{\xi}, 1)$, such that $\left|x-x^{\prime}\right| \geqslant \mu$. Hence, by a well known theorem, the series converges for all such values of $x$ and $x^{\prime}$ to the value $\frac{d}{d x^{\prime}} K\left(x, x^{\prime}\right)$.

Since $\Sigma \frac{\phi_{r}(x)}{\mu_{r}} \frac{\phi_{r}\left(x^{\prime}\right)}{r}$ converges uniformly in the interval $(\eta, 1)$ of $x^{\prime}$ to the value $K\left(x, x^{\prime}\right)$, a value $n_{2}$ of $n$ can be so chosen that

$$
\left|\int_{a^{\prime}}^{\beta^{\prime}}\left(\frac{m^{2}}{x^{\prime}}-\beta^{2}\right) K\left(x, x^{\prime}\right) d x^{\prime}-\int_{\alpha^{\prime}}^{\beta^{\prime}}\left(\frac{m^{2}}{x^{\prime}}-\beta^{2}\right) \sum_{r=1}^{r=n} \frac{\phi_{r}(x) \phi\left(x^{\prime}\right)}{\mu_{r}} d x^{\prime}\right|<\frac{1}{4} \epsilon
$$

for $n \geqslant n_{2}$, and for all values of $\alpha^{\prime}, \beta^{\prime}$ in the interval $(\eta, 1)$.

Also, a value $n_{3}$ of $n$ can be so chosen that

$$
\begin{aligned}
\mid 4 \beta^{\prime} & \sum_{r=1}^{r=n} \frac{\phi_{r}(x) \phi_{r}^{\prime}\left(\beta^{\prime}\right)}{\mu_{r}}-4 \alpha^{\prime} \sum_{r=1}^{r=n} \frac{\phi_{r}(x) \phi_{r}^{\prime}\left(\alpha^{\prime}\right)}{\mu_{r}} \\
& -4 \beta^{\prime} \frac{d}{d \beta^{\prime}} K\left(x, \beta^{\prime}\right)+4 \alpha^{\prime} \frac{d}{d \alpha} K\left(x, a^{\prime}\right) \mid<\frac{1 \epsilon}{}
\end{aligned}
$$


for all values of $a^{\prime}, \beta^{\prime}$ in the interval $(\eta, 1)$, and for all values of $x$ belonging to the interval $(\xi, 1)$, and not interior to the interval $\left(a^{\prime}-\mu, \beta^{\prime}+\mu\right)$, provided $n \geqslant n_{8}$.

If, now, $n_{1}$ denote the greater of the two integers $n_{2}, n_{3}$, we see that

$$
\int_{a^{\prime}}^{\beta^{\prime}} \sum_{r=1}^{r=n} \phi_{r}(x) \phi_{r}\left(x^{\prime}\right) d x^{\prime}
$$

differs from $\int_{a^{\prime}}^{\beta^{\prime}}\left(\frac{m^{2}}{x^{\prime}}-\beta^{2}\right) K\left(x, x^{\prime}\right) d x^{\prime}-4 \beta \frac{d}{d \beta^{\prime}} K\left(x, \beta^{\prime}\right)+4 a^{\prime} \frac{d}{d a^{\prime}} K\left(x, \alpha^{\prime}\right)$

by less than $\frac{1}{2} \epsilon$, if $n \geqslant n_{1}$.

The last expression is equal to

$$
\int_{a^{\prime}}^{\beta^{\prime}} \frac{d}{d x^{\prime}}\left\{4 x^{\prime} \frac{d}{d x^{\prime}} K\left(x, x^{\prime}\right)\right\} d x^{\prime}-4 \beta^{\prime} \frac{d}{d \beta^{\prime}} K\left(x, \beta^{\prime}\right)+4 a^{\prime} \frac{d}{d \boldsymbol{\alpha}} K\left(x, a^{\prime}\right)
$$

or to zero, since $K\left(x, x^{\prime}\right)$ satisfies the differential equation

$$
\frac{d}{d x^{\prime}}\left(4 x^{\prime} \frac{d y}{d x^{\prime}}\right)+\left(\beta^{2}-\frac{m^{2}}{x^{\prime}}\right) y=0 .
$$

It has thus been shewn that

$$
\left|\int_{a^{\prime}}^{\beta^{\prime}} \sum_{r=1}^{r=n} \phi_{r}(x) \phi_{r}\left(x^{\prime}\right) d x^{\prime}\right|<\frac{1}{2} \epsilon
$$

for $n \geqslant n_{1}$, and for all values of $\alpha^{\prime}, \beta^{\prime}$ in the interval $(\eta, 1)$, where $x$ is in the interval $(\xi, 1)$ and is not interior to the interval $\left(\alpha^{\prime}-\mu, \beta^{\prime}+\mu\right)$.

Therefore

$$
\left|\int_{a}^{\beta}\left(x x^{\prime}\right)^{ \pm} \sum_{r=1}^{r=n} \phi_{r}(x) \phi_{r}\left(x^{\prime}\right) d x^{\prime}\right|<\epsilon
$$

for all values of $\alpha$ and $\beta$ in the interval $(0,1)$, where $x$ is in $(\xi, 1)$ and not interior to the interval $(\alpha-\mu, \beta+\mu)$, provided $n \geqslant n_{1}$. Since $n_{1}$ is dependent only on $\epsilon$, the theorem relating to the values of $\int_{a}^{\beta} F\left(x, x^{\prime}, n\right) d x$ has been completely established, for the case in which the interval $(0,1)$ is the interval of definition of an arbitrary function, and in which the set $G$ consists of all the points of the interval $(\xi, 1)$, where $\hat{\xi}$ is an arbitrarily fixed positive number.

It follows, in virtue of the general convergence theorem, that, if $\chi(x)$ is any function which has a Lebesgue integral in the interval $(0,1)$, then

$$
\left.\int_{1}^{r-\mu} X\left(x^{\prime}\right)(x)^{\prime}\right)^{\frac{1}{r}} \sum_{r=1}^{n} \phi_{r}(x) \phi_{r}\left(x^{\prime}\right) d x
$$


converges, as $n$ is indefinitely increased, uniformly to zero, for all values of $x(>\mu)$ belonging to an interval $(\xi, 1)$, where $\xi>0$. Also

$$
\int_{x+\mu}^{1} \chi\left(x^{\prime}\right)\left(x x^{\prime}\right)^{\frac{1}{r=n}} \sum_{r=1}^{n} \phi_{r}(x) \phi_{r}\left(x^{\prime}\right) d x^{\prime}
$$

converges, as $n$ is indefinitely increased, uniformly to zero, for all values of $x$ in the interval $(\xi, 1)$ and not exceeding $1-\mu$.

This result holds whatever positive values may be assigned to the numbers $\mu, \xi$.

7. The theorem just established shews that the question of the convergence, as $n$ is indefinitely increased, of the integral

$$
\int_{0}^{1} \chi\left(x^{\prime}\right)\left(x x^{\prime}\right)^{\frac{1}{2}} \sum_{r=1}^{n} \phi_{r}(x) \phi_{r}\left(x^{\prime}\right) d x^{\prime}
$$

for any particular value of $x$ in an interval $(\xi, 1)$, can be answered by examining the nature of the function $\chi(x)$ in an arbitrarily small neighbourhood $(x-\mu, x+\mu)$ of the point $x$ concerned ; the function $\chi(x)$ being outside that neighbourhood only restricted by the condition that it shall have a Lebesgue integral in the whole interval $(0,1)$.

Now let $x^{\frac{1}{2}} \chi(x)=\Phi(x)$, and let us consider the integral

$$
\int_{0}^{1} \phi\left(x^{\prime}\right) \sum_{r=1}^{i=n} \phi_{r}(x) \phi_{r}\left(x^{\prime}\right) d x^{\prime}
$$

where $\Phi(x)$ is a function such that $x^{-\frac{1}{2}} \Phi(x)$ has a Lebesgue integral in the interval $(0,1)$.

The theorem established in $\S 6$ shews that

$$
\int_{0}^{x-\mu} \Phi\left(x^{\prime}\right) \sum_{r=1}^{r=n} \phi_{r}(x) \phi_{r}\left(x^{\prime}\right) d x^{\prime}, \quad \int_{x+\mu}^{1} \Phi\left(x^{\prime}\right) \sum_{r=1}^{r=n} \phi_{r}(x) \phi_{r}(x) d x
$$

converge uniformly to zero, as $n$ is indefinitely increased, for all values of $x$ in the interval $(\xi, 1)$; where also $x \geqslant \mu$ in the first integral, and $x \leqslant 1-\mu$ in the second integral.

It will now be shewn that the conditions stated in $\S \S 4,5$ (pp. 360-362, loc. cit.) of my paper on the general convergence theorem are satisfied by the function

$$
F\left(x^{\prime}, x, n\right)=\sum_{r=1}^{r=n} \phi_{r}(x) \phi_{r}\left(x^{\prime}\right) .
$$

It will first be shewn that

$$
\int^{x+\mu} \sum_{r=1}^{r=n} \phi_{r}(x) \phi_{r}\left(x^{\prime}\right) d x^{\prime}, \quad \int_{r-\mu}^{x} \sum_{r=1}^{r=n} \phi_{r}(x) \phi_{r}\left(x^{\prime}\right) d x^{\prime} .
$$


1908.] Representation of a FUnCtion By SERIES OF Bessel's functions. 379

where $\mu$ has a fixed value, converge uniformly to fixed values independent of $x$ for all values of $x$ in an interval interior to the interval $(\xi, 1)$.

We have, as in $\$ 6$ above,

$$
\begin{aligned}
\int_{x}^{x \pm \mu} \sum_{r=1}^{r=n} \phi_{r}(x) \phi_{r}\left(x^{\prime}\right) d x^{\prime}= & \int_{x}^{x \pm \mu}\left(\frac{m^{2}}{x}-\beta^{2}\right) \sum_{r=1}^{r=n} \frac{\phi_{r}(x) \phi_{r}\left(x^{\prime}\right)}{\mu_{r}} d x \\
& -4(x \pm \mu) \sum_{r=1}^{r=n} \frac{\phi_{r}(x) \phi_{r}^{\prime}(x \pm \mu)}{\mu_{r}}+4 x \sum_{r=1}^{r=n} \frac{\phi_{r}(x) \phi_{r}^{\prime}(x)}{\mu_{r}} .
\end{aligned}
$$

As before, the first term on the right-hand side converges uniformly to the value

where

$$
4 \beta^{\prime} \frac{d}{d \beta^{\prime}} K\left(x, \beta^{\prime}\right)-4 \alpha^{\prime} \frac{d}{d a^{\prime}} K\left(x, a^{\prime}\right)
$$

The series

$$
a^{\prime}=x+\beta^{\prime}=x+\mu \text {. }
$$

$$
\sum_{r=1}^{r=n} \frac{\phi_{r}(x) \phi_{r}^{\prime}(x+\mu)}{\mu_{r}}
$$

converges uniformly to the value $\frac{d}{d \beta^{\prime}} K\left(x, \beta^{\prime}\right)$, where $\beta=x+\mu$. There remains for consideration the series

This consists of a part

$$
\sum_{r=1}^{r=n} \frac{\phi_{r}(x) \phi_{r}^{\prime}(x)}{\mu_{r}} .
$$

$$
\frac{(-1)^{m}}{4 \pi x} \sum_{r=1}^{r=\pi} \frac{\cos 2(\kappa+r \pi) x^{b}}{r}
$$

which converges uniformly in an interval of $x$ interior to $(\hat{\xi}, 1)$, and of other parts which converge uniformly for all. values of $x$ in $(\xi, 1)$. It follows that $\sum_{r=1}^{r=n} \frac{\phi_{r}(x) \phi_{r}^{\prime}(x)}{\mu_{r}}$ converges uniformly to the value $\frac{1}{2} \frac{d K(x, x)}{d x}$. Hence

$$
\int_{x}^{x+\mu} \sum_{r=1}^{r=n} \phi_{r}(x) \phi\left(x^{\prime}\right) d x^{\prime}
$$

converges uniformly to the value

$$
4 x\left\{\frac{1}{2} \frac{d K(x, x)}{d x}-\frac{d}{d a^{\prime}} K\left(x, \alpha^{\prime}\right)\right\},
$$

where $a^{\prime}=x+0$. It will be shewn that this last expression is equal to $\frac{1}{2}$. For

$$
\frac{d}{d x} K(x, x)=\phi^{\prime}(x) \psi(x)+\phi(x) \psi^{\prime}(x),
$$

with the notation of $\S 4$; also

$$
\left\{\frac{d}{d \alpha^{\prime}} K\left(x, a^{\prime}\right)\right\}_{a^{\prime}=x+0}=\phi(x) \psi^{\prime}(x) ;
$$


and thus

$$
4 x\left[\frac{1}{2} \frac{d K(x, x)}{d x}-\left\{\frac{d}{d a^{\prime}} K\left(x, \alpha^{\prime}\right)\right\}_{a^{\prime}=x+0}\right]=2 x\left\{\phi^{\prime}(x) \psi(x)-\phi(x) \psi^{\prime}(x)\right\},
$$

and the expression on the right-hand side is, in accordance with $\S 4$, equal to $\frac{1}{2}$.

It has now been shewn that, for a fixed value of $\mu$,

$$
\int_{x}^{x+\mu} \sum_{r=1}^{r=n} \phi_{r}(x) \phi_{r}\left(x^{\prime}\right) d x^{\prime}
$$

converges to the value $\frac{1}{2}$, uniformly for all values of $x$ in an interval interior to the interval $(\xi, 1)$.

It may, in a precisely similar manner, be shewn that

$$
\int_{x-\mu}^{x} \sum_{r=1}^{r=n} \phi_{r}(x) \phi_{r}\left(x^{\prime}\right) d x^{\prime}
$$

converges uniformly to $\frac{1}{2}$ in a similar interval; $\mu$ being so chosen that $x-\mu$ is $>0$ in the interval of uniform convergence.

At the point $x=1$, we have to consider the limit of

$$
\int_{1-\mu}^{1} \sum_{r=1}^{r=n} \phi_{r}(x) \phi_{r}\left(x^{\prime}\right) d x^{\prime}
$$

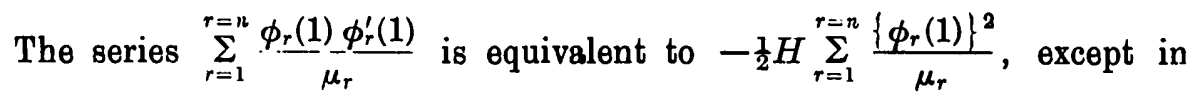
the cases $H=\infty, H=0$, when the series vanishes. We have then

$$
\begin{aligned}
\lim _{n=\infty} \int_{1-\mu}^{1} \sum_{r=1}^{r=n} \phi_{r}(1) \phi_{r}\left(x^{\prime}\right) d x^{\prime} \\
=4\left[\frac{d}{d \beta^{\prime}} K\left(1, \beta^{\prime}\right)\right]_{\beta^{\prime}=1}-4(1-\mu)\left[\frac{d}{d a^{\prime}} K\left(1, a^{\prime}\right)\right]_{a^{\prime}=1-\mu} \\
\quad-4 \sum_{r=1}^{r=\infty} \frac{\phi_{r}(1) \phi_{r}^{\prime}(1)}{\mu_{r}}+4(1-\mu) \sum_{r=1}^{r=\infty} \frac{\phi_{r}(1) \phi_{r}^{\prime}(1-\mu)}{\mu_{r}} \\
=4 \psi(1) \phi^{\prime}(1)+2 H K(1,1) \\
=4\left\{\psi(1) \phi^{\prime}(1)-\phi(1) \psi^{\prime}(1)\right\} \\
=1 .
\end{aligned}
$$

This result holds also in the case $H=0$. In the case $H=\infty$, it does not hold, but $\phi_{r}(1)$ is then zero, for all values of $r$, and the series of normal functions then converges to the value zero. 
1908.] Representation of a function by SERies of Bessel's functions. 381

8. Lastly, it will be shewn that the integrals $\int_{\mu_{1}}^{\mu} F(x \pm t, x, n) d t$ are, for a sufficiently small fixed value of $\mu$, both numerically less than fixed positive numbers, for all values of $\mu_{1}$ such that $0 \leqslant \mu_{1}<\mu$, and for all values of $x$ in an interval $\left(\xi^{\prime}, 1-\xi^{\prime \prime}\right)$; where $F\left(x^{\prime}, x, n\right)$ denotes the sum

$$
\sum_{r=1}^{r=n} \phi_{r}(x) \phi_{r}\left(x^{\prime}\right) \text {. }
$$

The number $\mu$ can be so chosen that $\xi^{\prime}>\mu, \xi^{\prime \prime}>\mu$.

In order to estimate the value of the integral $\int_{\mu_{1}}^{\mu} F(x+t, x, n) d t$, we employ the expression (7) of $\$ 3$, for $\phi_{r}(x+t)$, and we can consider separately the various terms of this expression. We have

$$
\begin{aligned}
& \int_{\mu_{1}}^{\mu} \frac{1}{(x+t)^{\frac{3}{2}}}{ }_{r=1}^{i=n} \cos \left\{\frac{m \pi}{2}+\frac{\pi}{4}-(\kappa+r \pi)(x+t)^{\frac{1}{3}}\right\} \phi_{r}(x) d t \\
& =(\mu+x)^{t} \int_{\mu_{2}}^{\mu} \frac{1}{(x+t)^{\frac{1}{2}}} \sum_{r=1}^{r=n} \cos \left\{\frac{m \pi}{2}+\frac{\pi}{4}-(\kappa+r \pi)(x+t)^{\frac{1}{4}}\right\} \phi_{r}(x) d t \\
& =(\mu+x)^{\frac{z}{r=m}} \sum_{r=1}^{r=1}\left[2 \sin \left\{\frac{m \pi}{2}+\frac{\pi}{4}-(\kappa+r \pi)(x+t)^{\frac{1}{2}}\right\}\right]_{\mu}^{\mu_{2}} \frac{\phi_{r}(x)}{\kappa+r \pi} . \\
& \frac{1}{\kappa+r \pi}=\frac{1}{r \pi}\left\{1+\frac{\alpha(r)}{r}\right\} \text {, }
\end{aligned}
$$

we see, on substitution of $\phi_{r}(x)$ as given by the form (7) of $\$ 3$, that this part of the sum consists partly of uniformly convergent series of which the $r$-th term contains the factor $1 / r^{2}$, and partly of terms involving series such as

$$
\sum_{r=1}^{r=n} \frac{1}{r} \sin \left\{\frac{m \pi}{2}+\frac{\pi}{4}-(\kappa+r \pi)\left(x+\mu_{2}\right)^{3}\right\} \cos \left\{\frac{m \pi}{2}+\frac{\pi}{4}-(\kappa+r \pi) x^{\frac{1}{4}}\right\},
$$

with a similar expression with $\mu$ instead of $\mu_{2}$.

This series is the sum of the two series

$$
\begin{aligned}
& \sum_{r=1}^{r=n} \frac{1}{2 r} \sin \left\{m \pi+\frac{1}{2} \pi-(\kappa+r \pi)\left[\left(x+\mu_{2}\right)^{3}+x^{k}\right]\right\}, \\
& \sum_{r=1}^{r=n}-\frac{1}{2 r} \sin \left\{(\kappa+r \pi)\left[\left(x+\mu_{2}\right)^{\frac{3}{2}}-x^{3}\right]\right\} .
\end{aligned}
$$

It is known that the series $\sum_{r=1}^{r=n} \frac{\cos u r \pi}{r}, \sum_{r=1}^{r=n} \frac{\sin u r \pi}{r}$ are uniformly convergent if $u$ is confined to an interval $\left(\eta, 2-\eta^{\prime}\right)$, where $\eta$ and $\eta^{\prime}$ are positive 
numbers. Since $\left(x+\mu_{2}\right)^{\frac{1}{3}}+x^{\frac{1}{2}}$ is between $2 \xi^{\prime \frac{k}{3}}$ and $\left(1-\xi^{\prime \prime}\right)^{\frac{1}{2}}+\left(1-\xi^{\prime \prime}+\mu_{2}\right)^{\frac{1}{3}}$, this condition is satisfied for the two series into which the first of the above series may be split up.

Again, it can be shewn* that the expression $\sum_{r=1}^{r=n} \frac{\sin (p+r) u}{r}$ is numerically less than a fixed positive number, independent of $n$ and $u$, If $u$ is in an interval $\left(0,2 \pi-\eta^{\prime \prime}\right)$. Since $\left(x+\mu_{2}\right)^{\frac{1}{2}}-x^{\frac{1}{2}}$ is in the interval $(0, \mu)$, it follows that the expression $(\beta)$ is less than a fixed positive number.

We have next to consider that portion of $\int_{\mu_{2}}^{\mu} F(x+t, x, n) d t$ which is

* To prove this, we see that, instead of the expression

we may consider $\begin{gathered}\sum_{r=1}^{r=n} \frac{\sin (p+r) u}{r}, \\ \sum_{r=1}^{r=n} \frac{\sin (p+r) u}{p+r},\end{gathered}$

since the difference of these expressions is a series which converges uniformly.

$$
\text { If } s_{n}(u) \text { denotes } \sum_{r=1}^{r=n} \frac{\sin (p+r) u}{r} \text {, }
$$

we have

$$
\frac{d s_{\mathrm{n}}(u)}{d u}=\frac{\sin \left(p+n+\frac{1}{2}\right) u-\sin \left(p+\frac{1}{2}\right) u}{2 \sin \frac{1}{2} u,} ;
$$

and therefore $s_{n}(u)=\frac{1}{2} \int_{0}^{\prime \prime} \frac{\sin \left(p+n+\frac{1}{2}\right) u}{\sin \frac{1}{2} u} d u-\frac{1}{2} \int_{0}^{u} \frac{\sin \left(p+\frac{1}{2}\right) u}{\sin \frac{1}{2} u} d u$

$$
\begin{aligned}
& =\int_{0}^{u} \frac{\sin \left(p+n+\frac{1}{2}\right) u}{u} d u-\int_{0}^{u} \frac{\sin \left(p+\frac{2}{2}\right) u}{u} d u+I_{n}(u)-I_{0}(u) \\
& =\int_{0}^{(p+n+1) u} \frac{\sin u}{u} d u-\int_{0}^{(p+1) u} \frac{\sin u}{u} d u+I_{n}(u)-I_{0}(u),
\end{aligned}
$$

where

$$
I_{n}(u)=\int_{0}^{n} \sin \left(p+n+\frac{1}{2}\right) u \frac{u-2 \sin \frac{1}{2} u}{2 u \sin \frac{1}{2} u} d u \text {. }
$$

On integration by parts, we have

$$
I(u)=-\frac{u-2 \sin \frac{1}{2} u}{2 u \sin \frac{1}{2} u} \frac{\cos \left(p+n+\frac{1}{2}\right) u}{p+n+\frac{1}{2}}+\int_{0}^{u} \frac{\cos \left(p+n+\frac{1}{2}\right) u}{p+n+\frac{1}{2}} \frac{4 \sin ^{2} \frac{1}{2} u-u^{2} \cos \frac{1}{2} u}{4 u^{2} \sin ^{2} \frac{1}{2} u} d u .
$$

If $u$ be confined to the interval $(0, b)$, where $b<2 \pi$, the two expressions

$$
\frac{u-2 \sin \frac{1}{2} u}{2 u \sin \frac{1}{2} u}, \frac{4 \sin ^{2} \frac{1}{2} u-u^{2} \cos \frac{1}{2} u}{4 u^{2} \sin ^{2} \frac{1}{2} u}
$$

are limited, and therefore

$$
I_{n}(u)<\frac{A}{p+n+\frac{1}{2}},
$$

where $A$ is a fixed number. Also $\int_{0}^{\lambda} \frac{\sin u}{u} d u$ is well known to be limited for all positive values of $\lambda$. Hence the result has been established. 
1908.] Representation of a function by series of Bessel's functions. $\mathbf{3 8 3}$

of the forms

$$
\int_{\mu_{1}}^{\mu} \frac{1}{(x+t)^{\frac{3}{r}}} \sum_{r=1}^{r=n}\left[\frac{\alpha(r, x, t)}{r} \sin \left\{\frac{\cos }{2}+\frac{\pi}{4}-(\kappa+r \pi)(x+t)^{\frac{1}{2}}\right\} \phi_{r}(x)\right] d t,
$$

and which is equivalent, on using the mean-value theorem, to

$$
(\mu+x)^{1} \int_{\mu_{2}}^{\mu} \frac{1}{(x+t)^{\frac{1}{r}}} \sum_{r=1}^{r=n}\left[\frac{\alpha(r, x, t)}{r} \sin \left\{\frac{m \pi}{2}+\frac{\pi}{4}-(\kappa+r \pi)(x+t)^{3}\right\} \phi_{r}(x)\right] d t .
$$

This expression may be integrated by parts, and we have then an expression containing $r(\kappa+r \pi)$ in the denominator of the general term. Remembering that $\alpha(r, x, t)$ and its differential coefficient are both limited for all values of $r$, and for all the values of $x$ and $t$ concerned, we see that this part of the integral satisfies the required condition. manner.

The case of $\int_{\mu_{1}}^{\mu} F(x-t, x, n) d x$ may be treated in a precisely similar

It can also be shewn that, except in the case $H=\infty$, for the point $x=1$, the condition is satisfied, that $\int_{\mu_{1}}^{\mu} F(1-t, 1, n) d t$ is numerically less than a fixed positive number, for all values of $\mu_{1}$ such that $0 \leqslant \mu_{1}<\mu$.

In this case the portion of the expression corresponding to $(\alpha)$ and $(\beta)$ is

$$
\sum_{r=1}^{r=n} \frac{1}{2 r} \sin \left\{\frac{m \pi}{2}+\frac{\pi}{4}-(\kappa+r \pi)\left(1-\mu_{1}\right)^{\frac{1}{2}}\right\} \cos \left\{\frac{m \pi}{2}+\frac{\pi}{4}-(\kappa+r \pi)\right\} .
$$

Except when $H=\infty$, we have

$$
\kappa=\frac{m \pi}{2}+\frac{\pi}{4}+s \pi
$$

therefore this expression may be written

$$
\sum_{r=1}^{r=1} \frac{1}{2 r} \sin \left\{(\kappa+r \pi)\left[1-\left(1-\mu_{1}\right)^{k}\right]\right\},
$$

and this is numerically less than a fixed positive number, independent of $r$ and $\mu$, for all values of $\mu_{1}<\mu$, as has been shewn above.

When $H=\infty$, we have

$$
\kappa=\frac{m \pi}{2}+\frac{3 \pi}{4}+s \pi,
$$

and the series takes the form

$$
\sum_{i=1}^{r=n} \frac{1}{2 r} \cos \left\{(\kappa+r \pi)\left[1-\left(1-\mu_{1}\right)^{k}\right]\right\},
$$


384

Dk. E. W. Hobson

[Dec. 10,

which is divergent when $\mu_{1}=0$, and therefore the expression is not limited.

The remaining part of $\int_{\mu_{1}}^{\mu} F(1-t, 1, n) d t$ may, as before, be shewn to be numerically less than fixed positive numbers.

9. It is now convenient to replace $x$ and $x^{\prime}$ by $z^{2}$ and $z^{\prime 2}$ respectively. The variables $z, z^{\prime}$ will be taken to be positive, and they are in the interval $(0,1)$.

If

we have

$$
\begin{aligned}
& \left|z-z^{\prime}\right| \geqslant \mu^{\frac{1}{2}}, \\
& \left|x-x^{\prime}\right| \geqslant \mu .
\end{aligned}
$$

It follows, from the result obtained in $\$ 5$, that

$$
\left|\left(z z^{\prime}\right)^{\underline{z}} \sum_{r=1}^{r=n} \phi_{r}\left(z^{2}\right) \phi_{r}\left(z^{\prime 2}\right)\right|
$$

is less, for all values of $n$, than a fixed positive number $N$, for all values of $z$ and $z^{\prime}$ in the interval $(0,1)$ which are such that $\left|z-z^{\prime}\right| \geqslant \mu^{\prime}$.

From another result in $\S 6$, it follows that

$$
\left|\int_{a}^{\beta}\left(z z^{\prime}\right)^{\frac{1}{2}} \sum_{r=1}^{r=n} \phi_{r}\left(z^{2}\right) \phi_{r}\left(z^{\prime 2}\right) z^{\prime} d z^{\prime}\right|<\frac{1}{2} \epsilon
$$

for all values of $\alpha, \beta$ in the interval $(0,1)$, and for all values of $z$ in an interval $\left(\xi^{\prime}, 1\right)$, and not interior to the interval $\left(\alpha-\mu^{\frac{k}{2}}, \beta+\mu^{\frac{1}{2}}\right)$; for $n \geqslant n_{1}$, where $n_{1}$ depends only on $\mu, \epsilon$, and $\xi^{\prime}$.

We have now, by employing the second mean-value theorem,

$$
\int_{a}^{\beta}\left(z z^{\prime}\right)^{\frac{3}{2}} \sum_{r=1}^{r=n} \phi_{r}\left(z^{2}\right) \phi_{r}\left(z^{\prime 2}\right) d z^{\prime}=\frac{1}{\alpha} \int_{a}^{\beta^{\prime}}\left(z z^{\prime}\right)^{\frac{1}{2}} \sum_{r=1}^{r=n} \phi_{r}\left(z^{2}\right) \phi_{r}\left(z^{\prime 2}\right) z^{\prime} d z^{\prime}
$$

where $a \leqslant \beta^{\prime} \leqslant \beta$. The expression on the right-hand side is numerically less than $\epsilon / 2 \alpha$, if the conditions stated above are satisfied. We have now

$$
\int_{\eta^{\prime}}^{\eta}\left|\left(z z^{\prime}\right)^{\frac{1}{2}} \sum_{r=1}^{r=n} \phi_{r}\left(z^{2}\right) \phi_{r}\left(z^{2}\right)\right| d z^{\prime}<N \eta
$$

if $z$ is not in the interval $\left(\eta^{\prime}-\mu^{\frac{1}{2}}, \eta+\mu^{b}\right)$. Let $\eta$ be now arbitrarily chosen ; then, if $\eta \leqslant \alpha$, we have

$$
\left|\int_{a}^{\beta}\left(z z^{\prime}\right)^{2} \sum_{r=1}^{r=n} \phi_{r}\left(z^{2}\right) \phi_{r}\left(z^{\prime 2}\right) d z^{\prime}\right|<\frac{\epsilon}{2 \eta}
$$


1908.] Representation of a function by series of Bessel's functions. 385

and, if $\alpha<\eta$, we have

$$
\left|\int_{a}^{\beta}\left(z z^{\prime}\right)^{\Downarrow} \sum_{r=1}^{r=n} \phi_{r}\left(z^{2}\right) \phi_{r}\left(z^{\prime 2}\right) d z^{\prime}\right|<\frac{\epsilon}{2 \eta}+N \eta,
$$

for $n \geqslant n_{1}$. Now, let $\epsilon^{\prime}$ be an arbitrarily small tixed number; we may then choose $\eta$ to be $\frac{1}{2} N^{-1} \epsilon^{\prime}$. We may then choose $n_{1}$ so great that $\epsilon$ is equal to $\frac{1}{2} N^{-1} \epsilon^{\prime 2}$; it then follows that

$$
\int_{a}^{\beta}\left(z z^{\prime}\right)^{\xi^{r}} \sum_{r=1}^{r=n} \phi_{r}\left(z^{2}\right) \phi_{r}\left(z^{2}\right) d z^{\prime}
$$

is numerically less than the arbitrarily chosen positive number $\epsilon^{\prime}$, provided $n \geqslant n_{1}$, for all values of $\alpha$ and $\beta$ in $(0,1)$, if $z$ is in the interval $\left(\xi^{\prime}, 1\right)$, and not interior to $\left(\alpha-\mu^{2}, \beta+\mu^{2}\right)$.

If, now, we let $F\left(z^{\prime}, z, n\right)$ denote the expression

$$
\left(z z^{\prime}\right)^{\frac{1}{d}} \sum_{r=1}^{r=n} \phi_{r}\left(z^{2}\right) \phi_{r}\left(z^{\prime 2}\right),
$$

we see that the conditions of the general convergence theorem are satisfied, the set $G$ to which $z$ is confined consisting of all the points of the interval $\left(\xi^{\prime}, 1\right)$. The number $\xi^{\prime}$ is arbitrarily small.

It follows that, for all values of $z$ in this interval $\left(\xi^{\prime}, 1\right)$,

$$
\begin{aligned}
& \int_{0}^{z-\mu^{2}} \chi\left(z^{\prime}\right)\left(z z^{\prime}\right)^{\frac{k}{2}} \sum_{r=1}^{r=n} \phi_{r}\left(z^{2}\right) \phi_{r}\left(z^{\prime 2}\right) d z^{\prime} \\
& \int_{-+\mu^{2}}^{1} \chi\left(z^{\prime}\right)\left(z z^{\prime}\right) \sum_{r=1}^{r-\sum^{\prime \prime}} \phi_{r}\left(z^{2}\right) \phi_{r}\left(z^{\prime 2}\right) d z^{\prime},
\end{aligned}
$$

converge uniformly to zero, where $\chi(z)$ denotes any function which has a Thebesgue integral in the interval $(0,1)$.

Now, let $\chi^{(z)}=z^{\ddagger} f(z)$; it then follows that the convergence of

$$
\int_{n}^{1} f\left(z^{\prime}\right) \stackrel{r-1}{\Sigma}_{r=1}^{\Sigma} \phi_{r}\left(z^{2}\right) \phi_{r}\left(:^{\prime 2}\right) z^{\prime} d ! !^{\prime}
$$

depends only on that of

$$
\int_{:=}^{:+\mu^{\prime}} f\left(z^{\prime}\right){ }_{r=1}^{r-n} \phi_{r}\left(z^{2}\right) \phi_{r}\left(z^{\prime 2}\right) z^{\prime} d z^{\prime}
$$

and of the similar integral with the limits $z, z-\mu^{\prime}$, the rest of the integral converging uniformly to zero, as $n$ is indefinitely increased, for all values of $z$ in the interval $\left(\xi^{\prime}, 1\right)$.

SkR. 2 . vol. 7 . No. 1029 . 
It has been shewn, in $\$ 8$, that

$$
\begin{aligned}
& \int_{z}^{z+\mu^{\prime}} \sum_{r=1}^{r=n} \phi_{r}\left(z^{2}\right) \phi_{r}\left(z^{2}\right) z^{\prime} d z^{\prime}, \\
& \int_{z-\mu^{\prime}}^{z} \sum_{r=1}^{r=n} \phi_{r}\left(z^{2}\right) \phi_{r}\left(z^{\prime 2}\right) z^{\prime} d z^{\prime},
\end{aligned}
$$

converge each to the value $\frac{1}{4}$, uniformly for all values of $z$ in the interval $\left(\xi^{\prime}, 1\right)$.

It has also been shewn that

$$
\int_{1-\mu^{\prime}}^{1} \sum_{r=1}^{r=n} \phi_{r}(1) \phi_{r}\left(z^{\prime 2}\right) z^{\prime} d z^{\prime}
$$

converges to the value $\frac{1}{2}$, as $n$ is indefinitely increased.

It has further been shewn that the integrals

$$
\int_{\mu_{1}}^{\mu} \sum_{r=1}^{r=n} \phi_{r}\left\{(z \pm t)^{2}\right\} \phi_{r}\left(z^{2}\right)(z \pm t) d t,
$$

are, for a sufficiently small fixed value of $\mu$, both numerically less than fixed positive numbers, for all values of $\mu_{1}$ such that $0 \leqslant \mu_{1}<\mu$, and for all values of $z$ in an interval $\left(\xi_{1}, 1-\xi_{2}\right)$.

It has also been shewn that, except when $K=\infty$,

$$
\left.\int_{\mu_{1}}^{\mu} \sum_{r=1}^{r=n} \phi_{r} \vdots(1-t)^{2}\right\} \phi_{r}(1)(1-t) d t,
$$

is numerically less than a fixed positive number, for all values of $\mu_{1}$ such that $0 \leqslant \mu_{1}<\mu$.

It follows, from the results established in $\$ \S 4,5$ of my earlier paper, that

$$
\int_{0}^{1} f\left(z^{\prime}\right) \sum_{r=1}^{r=n} \phi_{r}\left(z^{2}\right) \phi_{r}\left(z^{\prime 2}\right) z^{\prime} d z^{\prime}
$$

converges to the value $\frac{1}{4}\{f(z+0)+f(z-0)\}$,

for any interior point $z$ of the interval $(0,1)$, which is such that a neighbourhood of $z$ exists in which $f(z)$ has limited total fluctuation.

It also follows that the convergence to the value $\frac{1}{2} f(z)$ is uniform in any interval $(a, b)$ in which $f(z)$ is continuous, and which is interior to another interval in which $f(z)$ is of limited total fluctuation.

Also, at the point $z=1$, the integral converges to the value $\frac{1}{2} f(1-0)$ in case the point has a neighbourhood $(1-\eta, 1)$ in which the function is of limited total fluctuation. The only exception to this is in the case 


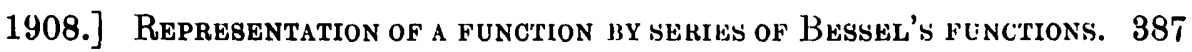
$K=\infty$, in which the integral vanishes for $z=1$. Remembering that

$$
\phi_{r}\left(z^{2}\right)=\frac{J_{m}\left(k_{r} z\right)}{\left\{\int_{0}^{1}\left[\left\{J_{m}\left(k_{r} z\right)\right\}^{2} 2 z d z\right]_{i}^{1 \frac{1}{2}}\right.},
$$

where $\lambda_{r}^{\frac{j}{2}}$ is here denoted by $k_{r}$, we have now established the following result.

Let $f(z)$ be any function defined for the interval $(0,1)$, and let it be such that, whether it be limited or not, in the interval, $z^{\mathrm{b}} f(z)$ has a Lebesgue integral in the interval $(0,1)$. Let $k_{1}, k_{2}, \ldots, k_{n}, \ldots$ denote the positive roots of the equation

$$
k J_{m l}^{\prime}(k)+H J_{m}(k)=0,
$$

where $m$ has any real value $\geqslant 0$, and $H$ is a constant which may have the value zero, in which case the equation is

$$
J_{m}^{\prime}(\lambda)=0 ;
$$

or $H$ may have the value infinity, in which case the equation is

$$
J_{n}(\lambda)=0 .
$$

The series

$$
\sum_{r=1}^{\infty} C_{r} J_{m}\left(k_{r} z\right) \int_{n}^{1} f\left(z^{\prime}\right) J_{n}\left(k_{r} z^{\prime}\right) z^{\prime} d z^{\prime},
$$

in which $C_{r}$ denotes

$$
\left[\int_{0}^{1}\left\{J_{n}\left(k_{r} z^{\prime}\right)_{i}^{\prime 2} z^{\prime} d z^{\prime}\right]^{-1}\right.
$$

converges to the value $\frac{1}{2}\{f(z+0)+f(z-0)\}$ at any point $z$ in the interior of the interval $(0,1)$ which is such that a neighbourhood of $z$ exists in which the function $f(z)$ is of limited total fluctuation (à variation bornée).

Moreover, in any interval $(a, b)$ in which $f(z)$ is continuous, and which is contained in the interior of another interval in which the function is of limited total fluctuation, the convergence of the series to the value $f(z)$ is uniform.

Except in the case $H=\infty$, the series converges at the point $z=1$ to the value $f(1-0)$, in case a neighbourhood $(1-\epsilon, 1)$ of that point exists in which the function is of limited total fuctuation. In the case $H=\infty$, the series converges to the value zero at the point $z=1$.

At the point $z=0$, the series converges to zero, except in the case $m=0$. In this case $m=0$, the convergence at the point $z=0$ has not been investigated. 
388 Representation of a function hy SERIES of Bessel's functions. [Dec. 10,

The value $k_{1}=0$ occurs among the values of $k_{r}$ only in the case $H=-m$, when the values of $k$, are the positive roots of the equation.

$$
J_{m+1}(k)=0 \text {. }
$$

In this case the first term of the series is

$$
2(m+1) z^{m} \int_{0}^{1} f\left(z^{\prime}\right) z^{\prime m+1} d z^{\prime}
$$

The value of the constant $C_{r}$ is $\frac{2 k_{r}^{2}}{\left[H^{2}+k_{r}^{2}-m^{2}\right] ! J_{m}\left(k_{r}\right)_{i}^{-2}}$, except when $H=\infty$, in which case $\quad C_{r}=\frac{2}{\zeta_{m}^{\prime}\left(k_{r}\right)_{i}^{\prime 2}}$.

When $H=-m, \quad C_{r}=\frac{2}{\left.\vdots J_{m}\left(k_{r}\right)\right\}^{\natural}}$.

It may be remarked that, in the special case in which $m=\frac{1}{2}, H=\infty$,

$$
J_{1}(z)=\left(\frac{2}{\pi}\right)^{\frac{1}{2}} \frac{\sin z}{z^{\frac{1}{2}}}
$$

the series becomes the l'ourier's sine-series after multiplication by $z^{\underline{z}}$, and replacing $z^{\sharp} f(z)$ by $f(z)$. 